\title{
STAR FORMATION IN W3-AFGL 333: YOUNG STELLAR CONTENT, PROPERTIES, AND ROLES OF EXTERNAL FEEDBACK
}

\author{
Jessy Jose ${ }^{1}$, Jinyoung S. Kim ${ }^{2}$, Gregory J. Herczeg ${ }^{1}$, Manash R. SAmal ${ }^{3}$, John H. Bieging ${ }^{2}$, \\ Michael R. Meyer ${ }^{4}$, AND William H. Sherry ${ }^{5,6}$ \\ ${ }^{1}$ Kavli Institute for Astronomy and Astrophysics, Peking University, Yi He Yuan Lu 5, Haidian Qu, Beijing 100871, China; jessyvjose1@gmail.com \\ ${ }^{2}$ Steward Observatory, University of Arizona, 933 North Cherry Avenue, Tucson, AZ 85721-0065, USA \\ ${ }^{3}$ Aix Marseille Université, CNRS, LAM (Laboratoire d'Astrophysique de Marseille) UMR 7326, F-13388 Marseille, France \\ ${ }^{4}$ Institute for Astronomy, ETH Zurich, Wolfgang-Pauli-Strasse 27, 8093 Zurich, Switzerland \\ ${ }^{5}$ National Optical Astronomy Observatories, 950 North Cherry Avenue, Tucson, AZ 87719, USA \\ ${ }^{6}$ Eureka Scientific, Inc. 2452 Delmer Street Suite 100, Oakland, CA 94602-3017, USA \\ Received 2015 September 20; accepted 2016 February 16; published 2016 May 3
}

\begin{abstract}
One of the key questions in the field of star formation is the role of stellar feedback on the subsequent star formation process. The $\mathrm{W} 3$ giant molecular cloud complex at the western border of the $\mathrm{W} 4$ super bubble is thought to be influenced by the massive stars in W4. This paper presents a study of the star formation activity within AFGL 333, a $\sim 10^{4} M_{\odot}$ cloud within W3, using deep $J H K_{s}$ photometry obtained from the NOAO Extremely Wide Field Infrared Imager combined with Spitzer IRAC and MIPS photometry. Based on the infrared excess, we identify 812 candidate young stellar objects (YSOs) in the complex, of which 99 are Class I and 713 are Class II sources. The stellar density analysis of YSOs reveals three major stellar aggregates within AFGL 333, namely AFGL 333 Main, AFGL 333 NW1 and AFGL 333 NW2. The disk fraction within AFGL 333 is estimated to be $\sim 50 \%-60 \%$. We use the extinction map made from the $H-K_{s}$ colors of the background stars and CO data to understand the cloud structure and to estimate the cloud mass. From the stellar and cloud mass associated with AFGL 333, we infer that the region is currently forming stars with an efficiency of $\sim 4.5 \%$ and at a rate of $\sim 2-3 M_{\odot}$ $\mathrm{Myr}^{-1} \mathrm{pc}^{-2}$. In general, the star formation activity within AFGL 333 is comparable to that of nearby low mass star-forming regions. We do not find any strong evidence to suggest that the stellar feedback from the massive stars of nearby W4 super bubble has affected the global star formation properties of the AFGL 333 region.
\end{abstract}

Key words: stars: formation - stars: pre-main sequence

Supporting material: machine-readable table

\section{INTRODUCTION}

Most stars form in OB associations where stellar winds, UV radiation, and supernova explosions from the massive members significantly affect their environment. Feedback from newly born massive stars plays an active role in the subsequent evolution of their parent molecular clouds. Feedback can trigger the birth of new generations of stars that would otherwise not exist, allowing star formation to propagate continuously from one point to the next (Elmegreen \& Lada 1977). On the other hand, feedback may also inhibit star formation by clearing away the dust and gas (Bisbas et al. 2011; Dale et al. 2013; Walch et al. 2013).

Numerical simulation studies by Dale \& Bonnell (2012) and Dale et al. (2013) show that the effect of feedback in a massive star-forming region depends on its cloud mass and density. The most massive and largest clouds are mostly dynamically unaffected by stellar feedback. On the other hand, feedback has a profound effect on the lower density clouds, expelling tens of percent of the neutral gas long before any massive star explodes as a supernova. Since giant molecular clouds typically have complicated, clumpy, internal structures, the stellar feedback mechanisms penetrate into different depths in different directions of a given cloud, producing highly irregular morphologies (Walch et al. 2013). The global influence of feedback on a given system may therefore differ from the local effects; star formation could be suppressed at some locations and triggered in others (Dale \& Bonnell 2012).
The W3 star-forming complex ( $d \sim 2 \mathrm{kpc}$; Hachisuka et al. 2006; $\mathrm{Xu}$ et al. 2006), one of the most massive molecular clouds in the outer Galaxy $\left(\sim 4 \times 10^{5} M_{\odot}\right.$; Moore et al. 2007 ; Polychroni et al. 2012), has long been proposed as a classic example of induced or triggered star formation (Lada et al. 1978; Oey et al. 2005). The W3 complex has a complicated structure. The pressure from the expanding $\mathrm{H}$ II region and stellar winds from the massive stars of the W4 super bubble have been suggested to have swept up the molecular cloud to create the "high density layer" (HDL) at its western periphery (Lada et al. 1978 and references therein). W3 Main, W3 (OH), W3 North, IC 1795, and AFGL 333 are the most active star-forming sites identified within the high density layer. Feedback from W4 was identified as a key factor for inducing and enhancing the star formation activity within the high density layer. Localized triggering from IC 1795 has been suggested to influence its surrounding regions such as W3 Main and W3 $(\mathrm{OH})$, whereas, to the west of the HDL (e.g., KR140), a spontaneous or quiescent mode of star formation has been suggested often (e.g., Kerton et al. 2008; Rivera-Ingraham et al. 2011, 2013; Kiminki et al. 2015). The most prominent regions within W3 are marked in Figure 1. Of these, W3 Main is the most active star-forming region, with more than 10 $\mathrm{H}$ II regions of various evolutionary status (Tieftrunk et al. 1997; Ojha et al. 2004). The scenario called "convergent constructive feedback" proposed by Rivera-Ingraham et al. $(2013,2015)$ suggests that star formation activity toward W3 


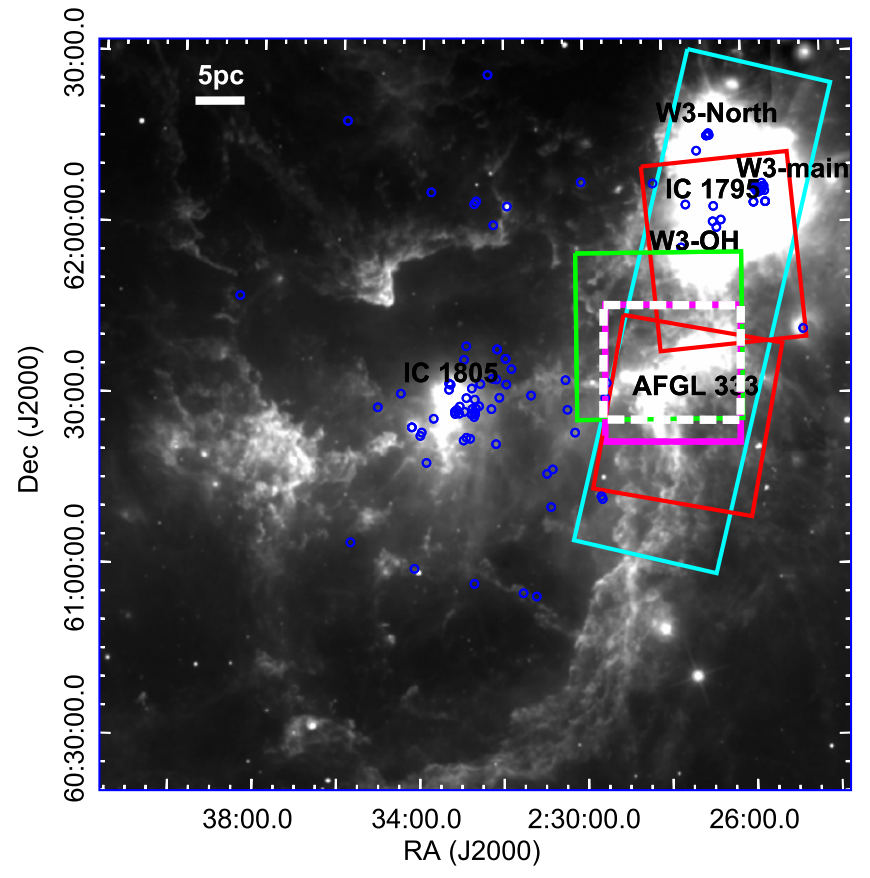

Figure 1. WISE $22 \mu \mathrm{m}$ wide-field image $\left(\sim 2^{\circ} \times 2^{\circ}\right)$ covering the W4 super bubble centered at the cluster IC 1805 along with the W3 GMC to the west. Locations of the important sub-regions of the W3 complex are marked and the boxes represent the area covered for various observations (green: NEWFIRM; red: IRAC; cyan: MIPS). Magenta box shows the area $\left(\sim 24^{\prime} \times 24^{\prime}\right)$ corresponding to the AFGL 333 region, based on the low resolution ${ }^{12} \mathrm{CO}$ ( $J=3-2)$ map by Sakai et al. (2006) and the white dashed box represents the area considered in this study. The blue circles denote the locations of the probable massive members (earlier than B3V) in the complex obtained from the SIMBAD database (http://simbad.u-strasbg.fr/simbad/).

Main is a self-enhancing process, where the youngest and most massive stars are observed at the innermost regions.

Within the W3 complex, the AFGL 333 region $\left(\alpha_{2000}=02^{\mathrm{h}} 28^{\mathrm{m}} 15^{\mathrm{s}} ; \delta_{2000}=+61^{\circ} 20^{\prime} 58^{\prime \prime}\right)$ lies in the southern part of the HDL, $\sim 17$ pc away from the center of W4 bubble (see Figure 1). AFGL 333 consists of (1) a bright rimmed cloud (BRC 5, Sugitani et al. 1991) associated with IRAS $02252+6120$, pointing directly to the massive stars of W4, (2) a H II region ionized by a B0.5 star (Hughes \& Viner 1982) associated with IRAS $02245+6115$, (3) a prominent dense filamentary structure associated with a molecular ridge (defined as AFGL 333 Ridge; Rivera-Ingraham et al. 2013), and several IRAS sources (see Figure 2). The morphology of this complex with BRC 5 facing the W4 OB association strongly suggests a large-scale feedback due to the expansion of W4. The projected distance between AFGL 333 and the massive stars in W4 is smaller when compared to that of the distance between W3 Main and W4. Hence the amount of stellar radiation and wind energy received at the surface of AFGL 333 Main may be higher than that of W3 Main.

The high density layer of the W3 complex has a diverse density structure, where W3 Main has the highest density compared to AFGL 333 (Rivera-Ingraham et al. 2015). Is the outcome of the star formation process, such as star formation efficiency and star formation rate (SFR), different in AFGL 333 and W3 Main? Stars form in both regions, but from gas with different densities subjected to different radiation environments. AFGL 333 seems to be externally influenced by W4, whereas the local triggering effect from the cluster IC 1795 and the internal self-enhancing process have been

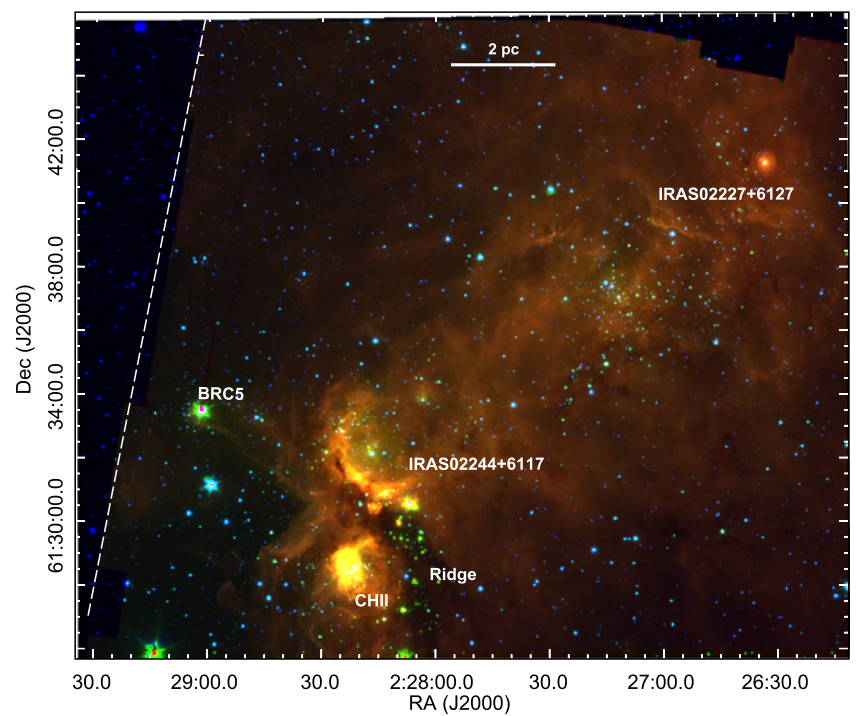

Figure 2. Color composite image of the $\sim 24^{\prime} \times 20^{\prime}$ area considered in this study (centered at $\alpha_{2000}=02^{\mathrm{h}} 27^{\mathrm{m}} 48^{\mathrm{s}} ; \quad \delta_{2000}=+61^{\circ} 35^{\prime} 58^{\prime \prime}$ ) made using $2.1 \mu \mathrm{m}$ (blue), $4.5 \mu \mathrm{m}$ (green), and $8.0 \mu \mathrm{m}$ (red) images. The important subregions are marked and the area to the left of the dashed line is not covered by 4.5 and $24 \mu \mathrm{m}$ bands.

given as the explanation for the high star formation activity within W3 Main (Oey et al. 2005; Rivera-Ingraham et al. 2013). In order to understand the nature of the YSO population and star formation activities in the sub-regions of W3, under different external conditions, we explore the stellar properties of this complex. The stellar content of AFGL 333both low and high mass-is less explored than W3 Main/OH. The latest census of the young stellar population of the W3 complex by Rivera-Ingraham et al. (2011) used shallow 2MASS and Spitzer observations. Since the initial mass function (IMF) of a star-forming region peaks toward the low mass stars, the star formation process is best traced by the identification of the low mass stellar population. Deep near-IR (NIR) and mid-IR (MIR) photometry are ideal tools to uncover the low mass stellar content of heavily obscured and dense environments.

In this paper, we use the deep NIR data in combination with Spitzer data to identify and characterize the young stellar objects (YSOs) within AFGL 333 as well as to understand the star formation activity of the region. The data sets presented in this study are unique in terms of its depth and completeness compared to other surveys of this region. The paper is organized as follows: Section 2 discusses the various data sets and photometry catalogs obtained. Sections 3 and 4 present detailed analysis of the extinction map and selection procedures of YSO candidates in AFGL 333. Various characteristics of YSOs and the newly identified stellar aggregates within AFGL 333 and their properties are discussed in Section 5. In Section 6 we compare our results with W3 Main as well as with various nearby low and high mass star-forming regions. We also discuss the implications of triggered star formation in AFGL 333. The results are summarized in Section 7.

\section{OBSERVATIONS AND POINT SOURCE CATALOGS}

\subsection{NEWFIRM NIR Imaging and Photometry}

We obtained NIR observations of AFGL 333 in $J H K_{s}$ bands using the National Optical Astronomical Observatory (NOAO) 
Extremely Wide Field InfraRed Imager (NEWFIRM; Probst et al. 2004) camera on the $4 \mathrm{~m}$ Mayal telescope at Kitt Peak National Observatory (KPNO) on 2009 December 6. The NEWFIRM camera contains 4 InSb $2048 \times 2048$ pixel arrays arranged in a $2 \times 2$ pattern with a field of view of $28^{\prime} \times 28^{\prime}\left(16.2 \times 16.2 \mathrm{pc}^{2}\right.$ at $\left.d=2 \mathrm{kpc}\right)$ and a pixel scale of 0 ". $4(0.0039 \mathrm{pc})$ per pixel. The area coverage of this observation is shown by a green box in Figure 1. We took 50,48 , and 72 second exposures for $J, H$, and $K_{s}$ bands, respectively, at nine dithered positions. The dither offsets were large enough to fill in the $1^{\prime}$ gap between CCDs. During our observations, the seeing was around $\sim 1$ "! $0-1$ "! 2 . Standard processing of dark frame subtraction, flat fielding, sky subtraction, and bad pixel masking was performed by the NEWFIRM Science Pipeline (Swaters et al. 2009) and final stacked images were produced in each band.

We used the DAOFIND task in IRAF to extract the preliminary list of point sources in the $K$-band image. This algorithm provides additional statistics of the point sources such as roundness and sharpness. In order to avoid the artifacts and false detections, we selected only those sources having $\mathrm{S} / \mathrm{N}>$ 5. Following Stetson (1987), we set the roundness limits as -1 to +1 and sharpness limits as 0.2 to +1 , which should eliminate the bad pixel brightness enhancements and the extended sources such as background galaxies from the point source catalog. Due to the non-uniform background nebular emission as well as crowdedness of the field, some sources were missed in the automatic detection algorithm. Such sources were manually identified and added in the final source list if they satisfy the above signal-to-noise ratio $(\mathrm{S} / \mathrm{N})$, roundness, and sharpness criteria. The list was again visually checked for any spurious source detection and such sources were deleted from the list. The astrometry has been corrected with respect to the Two Micron All Sky Survey (2MASS) point source catalog and the astrometry accuracy of our final source list is $\sim 0$ !" 3 .

The final source list was fed into the DAOPHOT ALLSTAR routine to obtain the point-spread function (PSF) photometry in $J, H$, and $K_{s}$ bands. Absolute photometric calibration was obtained using the 2MASS data (Skrutskie et al. 2006), with a quality flag better than "A" in all three bands. We matched up bright isolated stars from our NEWFIRM catalogs with sources from 2MASS within a radius of 1"! 0 and obtained zero points. The rms scatter between the calibrated NEWFIRM and 2MASS data (i.e., 2MASS-NEWFIRM data) for the $J, H$, and $K_{s}$ bands were $0.06,0.06$, and $0.07 \mathrm{mag}$, respectively. The saturated point sources in the NEWFIRM catalog were replaced by 2MASS magnitudes. We finally created the $J H K_{s}$ photometry catalog by spatially matching the detected sources in three bands within a match radius of 1 ". 0 . Only those sources that satisfy our reliability criteria in all three bands (i.e., photometry uncertainty $<0.2 \mathrm{mag}$ ) were included in the final list. The total number of sources detected in each band and the detection limits are listed in Table 1. Compared to the 2MASS completeness limits in the $J H K_{S}$ bands (i.e., 15.9, 15.1, and $14.3 \mathrm{mag}$ ), the new photometry is deeper by $4.6,3.6$, and 3.7 mag in $J, H$, and $K_{s}$ bands, respectively, and thus adding 10444,10624 , and 11441 more sources than the existing 2MASS catalog.

\subsection{Spitzer IRAC and MIPS Imaging and Photometry}

Images from the Spitzer Space Telescope (Werner et al. 2004) using the Infrared Array Camera (IRAC, Fazio
Table 1

Point Source Catalog Summary for Various Bands

\begin{tabular}{lcccc}
\hline \hline Band & $\begin{array}{c}\text { Sources in } \\
\text { Total Area }^{\text {a }}\end{array}$ & $\begin{array}{c}\text { Within } \\
\text { AFGL 333 }\end{array}$ & $\begin{array}{c}\text { Detection } \\
\text { Limit (mag) }\end{array}$ & $\begin{array}{c}\text { 90\% Completeness } \\
\text { Limit (mag) }\end{array}$ \\
\hline$J$ & 13162 & 7194 & 20.5 & 18.0 \\
$H$ & 13472 & 7447 & 18.7 & 17.5 \\
$K_{s}$ & 13573 & 7525 & 18.0 & 17.0 \\
ch1 & 22361 & 5346 & 17.8 & 16.0 \\
ch2 & 20502 & 6095 & 17.5 & 16.0 \\
ch3 & 4452 & 1408 & 15.3 & 13.0 \\
ch4 & 2400 & 799 & 14.0 & 12.0 \\
{$[24]$} & 327 & 134 & 9.3 & 7.0 \\
\hline
\end{tabular}

Notes.

${ }^{a}$ Number of sources within the field of view of individual bands.

${ }^{\mathrm{b}}$ Number of sources within AFGL 333 area considered in this study.

et al. 2004) centered at 3.6, 4.5, 5.8, and $8.0 \mu \mathrm{m}$ (ch1, ch2, ch3, and ch4) were obtained from the Spitzer archive. ${ }^{7}$ These observations were taken on 2004 January 10 and 2007 February 19 (Program IDs: 30955, 127; PIs: R. Gehrz, T. Moore) in high dynamic range (HDR) mode with three dithers per map position and two images each with an integration time of 0.4 and $10.4 \mathrm{~s}$ per dither. Preliminary analyses of these data sets have been presented in Ruch et al. (2007) and RiveraIngraham et al. (2011). The total area coverage by these two observations is $\sim 28^{\prime} \times 63^{\prime}\left(\sim 16 \times 37 \mathrm{pc}^{2}\right.$; see Figure 1).

We obtained the $\mathrm{cBCD}$ (corrected basic calibrated data) images (version S18.7.0) from the archive and the raw data were processed and calibrated with the IRAC pipeline. The final mosaic images were created using the MOPEX pipeline (version 18.0.1) with an image scale of 1 "' 2 per pixel. In order to avoid the saturation due to bright nebular emission, we processed the long and short exposure frames separately. We kept the settings similar to the NEWFIRM data (see Section 2.1) to make a preliminary source list using DAOFIND in IRAF. Since the IRAC bands suffered from variable background nebular emission over small spatial scales, a single point-source detection threshold across the entire mosaic image does not detect all the potential point sources in the field. We used various detection threshold values over multiple iterations to enable the detection of all faint sources in the field. The reliability of the sources was decided based on their $\mathrm{S} / \mathrm{N}$, roundness, and sharpness values. Many spurious sources in the nebulosity were identified by visual inspection and deleted from the automated detection list.

To extract the flux of the point sources, we performed point response function (PRF) fitting on IRAC images in multi-frame mode, using the Astronomical Point Source EXtraction (APEX) tool, developed by the Spitzer Science Center (see Jose et al. 2013 for details). Flux densities were converted into magnitudes using the zero points $280.9,179.7,115.0$, and 64.1 Jys in the 3.6, 4.5, 5.8, and $8.0 \mu \mathrm{m}$ bands, respectively, following the IRAC Data Handbook. ${ }^{8}$ The saturated bright sources in the long integrated images were replaced by the sources from short exposure images. To ensure optimal photometry, only those sources with $\mathrm{S} / \mathrm{N}>5$ and photometry uncertainty $<0.2 \mathrm{mag}$ in individual bands are considered for further analysis. The number of sources detected in each band

\footnotetext{
http://archive.spitzer.caltech.edu/

8 http://ssc.spitzer.caltech.edu/irac/iracinstrumenthandbook/
} 
and the detection limits are given in Table 1. The IRAC data of the four band passes were merged by matching the coordinates using a radial matching tolerance of 1 "' 2 . Thus our final IRAC catalog contains photometry of 29,224 sources that are detected in one or more IRAC bands, including 1567 sources detected in all four IRAC bands.

AFGL 333 was observed in $24 \mu \mathrm{m}$ using the Multi-band Imaging Photometer for Spitzer (MIPS; Rieke et al. 2004) on 2004 February 03 (Program ID: 127, PI: R. Gehrz). The observation covers an area of $\sim 26^{\prime} \times 89^{\prime}\left(\sim 15 \times 52 \mathrm{pc}^{2}\right.$, see Figure 1). We obtained the BCD images (S18.13.0) from the Spitzer archive and the final mosaics were created using the MOPEX pipeline (version 18.0.1) with an image scale of 2!" 45 per pixel. We applied the same source detection technique and reliability criteria as described for IRAC to the MIPS $24 \mu \mathrm{m}$ image. To extract the flux, we performed the PRF fitting method in single-frame mode. The zero-point value of $7.14 \mathrm{Jy}$ from the MIPS Data Handbook ${ }^{9}$ has been used to convert flux densities to magnitudes. The final catalog contains the $24 \mu \mathrm{m}$ photometry of 327 sources having an uncertainty $<0.2 \mathrm{mag}$.

\subsection{Completeness Limits}

In order to analyze the photometric incompleteness, the artificial star experiment has been performed using the ADDSTAR routine in IRAF on the $K_{s}$-band image (see Jose et al. 2012 for details). Briefly, artificial stars were added to the $K_{s}$-band image at randomly generated positions. The luminosity distribution of artificial stars was chosen in such a way that a greater number of stars was inserted toward the fainter magnitude bins. The frames were reduced using the same procedure used for the original frames (Section 2.1). The ratio of the number of stars recovered to those added in each magnitude interval gives the completeness factor as a function of magnitude. The faintest magnitude bin, where the fraction of sources recovered was greater than $90 \%$, was adopted as the completeness limit. We thus obtained $\sim 90 \%$ completeness of the data for the magnitude limit of 17 in $K_{s}$ band. The completeness limits of individual bands were also analyzed using the histogram distributions of the measured source magnitudes (see Figure 3; Table 1). The turnover point in source count curves can serve as a proxy to show the completeness limit (e.g., Jose et al. 2013; Willis et al. 2013; Samal et al. 2015). The photometric completeness obtained from histogram analysis is consistent with that estimated from the artificial star experiment in $K_{s}$ band.

\subsection{Final Point Source Catalog}

We created the final photometry catalog by spatially matching and merging the detected sources in various bands. A search for the IRAC counterparts of the NIR sources within a match radius of 1 !' 2 identified at least 7168 sources in one of the IRAC bands. Of the 327 detections in the MIPS band, within a match radius of 2 "! 5,304 sources have IRAC counterparts in at least one band, 135 sources with NIR counterparts.

We compared our IRAC and MIPS photometry with the existing catalogs in the literature (Ruch et al. 2007; RiveraIngraham et al. 2011). The root mean square (rms) scatter between the photometry from this work and that of Rivera-

\footnotetext{
http://ssc.spitzer.caltech.edu/mips/mipsinstrumenthandbook/
}

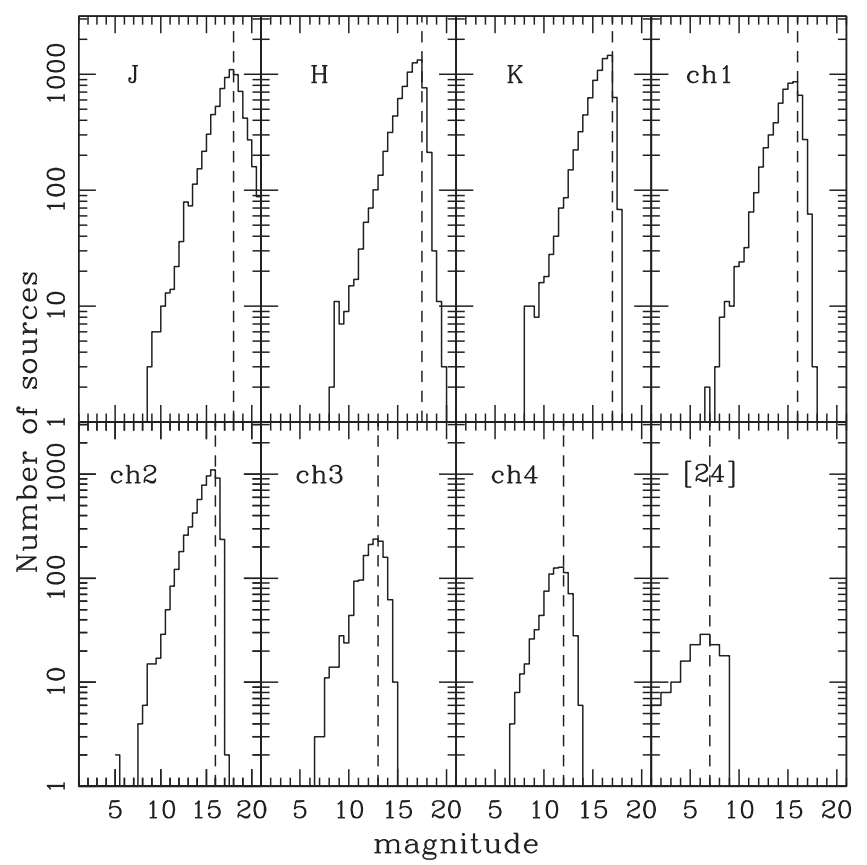

Figure 3. Source histograms for bands $J$ through $24 \mu \mathrm{m}$ showing the limiting magnitude and completeness limit for each band. The vertical lines indicate the adopted completeness limit.

Ingraham et al. (2011) are found to be within $0.1 \mathrm{mag}$ and are within 0.2 mag of that of Ruch et al. (2007) for all IRAC bands. This dispersion occurs mainly due to the different techniques adopted for the flux estimation, however, is within the uncertainty limit in this study. The rms scatter between the MIPS $24 \mu \mathrm{m}$ photometry of this study with that of Ruch et al. (2007) and Rivera-Ingraham et al. (2011) is $~ 0.5 \mathrm{mag}$.

Table 1 lists the number of sources detected in each band as well as their detection and completeness limits. The number of sources in each band is different due to respective sensitivity limits and area coverage (see Figure 1). The AFGL 333 region is projected on the sky over an area of $\sim 24^{\prime} \times 24^{\prime}$ (see Figure $1 ; \sim 193 \mathrm{pc}^{2}$ ), based on CO emission maps from Sakai et al. (2006) and Bieging \& Peters (2011). However, the coverage areas of the near-IR, IRAC, and MIPS observations are all different and do not cover the entire region (see Figure 1). An area $\left(\sim 24^{\prime} \times 20^{\prime}, 161 \mathrm{pc}^{2}\right)$ covered by most of the wavelengths is considered for this analysis (see Figure 2 and the white box in Figure 1). Our coverage area for extinction maps (Section 3) and our YSO identification (Section 4) miss $\sim 15 \%$ of the AFGL 333 area to the south compared with the area delineated by Sakai et al. (2006). The number of sources detected within the area of interest in each band is given in column 3 of Table 1.

\section{MAPPING THE DUST AND GAS IN AFGL 333}

The main goal of this study is to obtain the census of YSOs as a tool to understand the cloud structure and star formation activity within the AFGL 333 region. The census of YSOs based on color-color cuts requires a quantification of how color-color selections are affected by extinction. In this section, we map the structure of gas and dust in the AFGL 333 molecular cloud. These extinction maps also allow us to identify the sites of active star formation. 


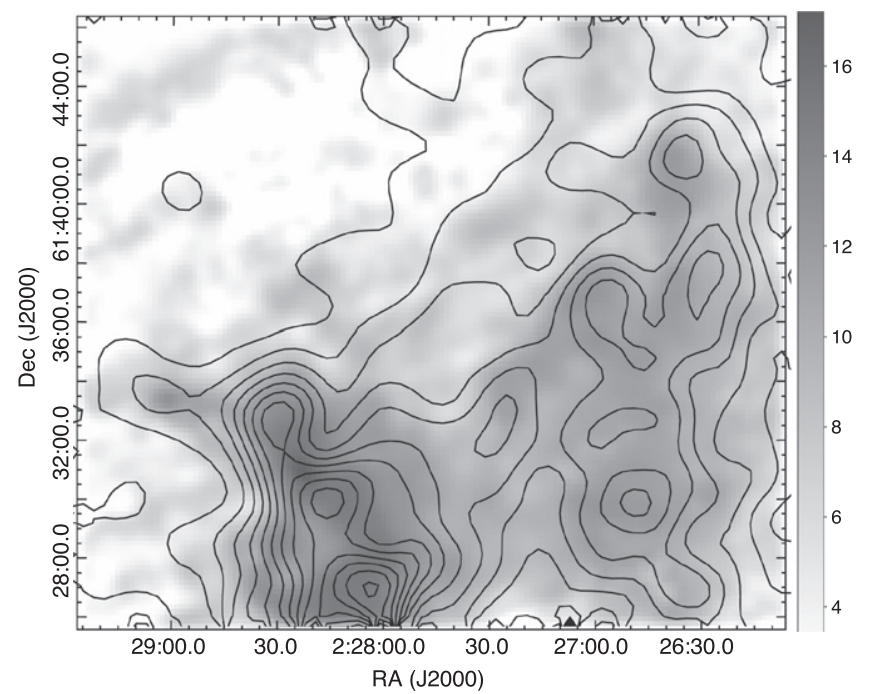

Figure 4. Grayscale represents the $V$-band extinction map made from the $\mathrm{CO}$ column density map and the contours are the $V$-band extinction map generated from the $\left(H-K_{s}\right)$ colors using the nearest neighborhood method assuming $R_{V}=3.1$. The contours begin at $A_{V}=4 \mathrm{mag}$ and increase by $1.5 \mathrm{mag}$ to a maximum of $25 \mathrm{mag}$.

The primary dust map used in this paper is a map of extinction calculated from near-IR colors of background stars (Section 3.1). In Section 3.2, we create a map of molecular gas from $1.3 \mathrm{~mm} \mathrm{CO}$ emission. In Section 3.3, the near-IR extinction map is then compared to the $\mathrm{CO}$ map and a published dust column density map calculated from emission in Herschel imaging to confirm consistency within the uncertainties between the three methods.

\subsection{Near-IR Extinction Maps of AFGL 333}

The dust column density may be mapped by calculating the extinction to background sources. Our extinction map is calculated by dereddening the $H-K_{s}$ of background stars to the nominal average intrinsic $H-K_{s}$ of field stars, i.e., $A_{K}=1.82 \times\left(H-K_{s}\right)_{\text {obs }}-\left(H-K_{s}\right)_{\text {int }}$, where $\left(H-K_{s}\right)_{\text {int }}=$ 0.2 is considered the average intrinsic color of field stars (Allen et al. 2008; Gutermuth et al. 2009; Jose et al. 2013).

Since AFGL 333 is situated at a distance of $\sim 2.0 \mathrm{kpc}$, an average foreground extinction of $A_{V}=2.6 \mathrm{mag}$ is expected (0.15 mag kpc ${ }^{-1}$ in $K_{S}$ band, Indebetouw et al. 2005; Chavarría et al. 2008; $A_{K} / A_{V}=0.114$; Cardelli et al. 1989). This value is consistent with the minimum interstellar reddening obtained toward W3 by Oey et al. (2005) and also with the all-sky dust map based on PAN-STARRS and 2MASS photometry by Green et al. (2015). Thus in order to eliminate the foreground contribution, only those stars with $A_{V}>2.6 \mathrm{mag}$ are used to obtain the extinction map. To generate the extinction map, the method described by Gutermuth et al. (2005) is followed. Briefly, the region is divided into uniform grids and the mean and standard deviation of $A_{V}$ values of $N$ nearest neighbor stars from the center of each grid was measured. The algorithm rejects any stars with $A_{V}$ values $>3 \sigma$ from the mean. The outlier rejection in this application should primarily remove the YSOs with IR excess, where $H-K_{s}$ excess mainly arises from their circumstellar disk (Meyer et al. 1997). The resulting $A_{V}$ map is convolved with a Gaussian kernel to get the final mean value. The final extinction map is generated with $N=6$ and angular resolution of $20^{\prime \prime}$ (Figure 4) after several iterations to achieve a good compromise between resolution and noise (Gutermuth et al. 2009).

The average value of $A_{V}$ across AFGL 333 is found to be $\sim 10$ mag. The extinction map of AFGL 333 given in Figure 4 shows two distinct features: a cavity with low extinction $\left(A_{V}<3.5 \mathrm{mag}\right)$ toward the eastern side of the image and a highly extincted western half. A comparison with the submillimeter and $\mathrm{C}^{18} \mathrm{O}(J=1-0)$ observations (see Sakai et al. 2006; Moore et al. 2007) shows that the highest extinction areas coincide with the dense molecular cores detected toward AFGL 333, including the curved features of BRC 5 and the AFGL 333 Ridge.

The uncertainty in the extinction measurement is dominated by the systematic error in the adopted extinction law, assumed here to be based on a total-to-selective extinction $R_{V}=3.1$ typical of the diffuse interstellar medium. However, dense clouds usually have $R_{V}$ values $>4-5.5$, especially at $A_{V}>20$ mag (Mathis 1990; Chapman et al. 2009), which would lead to a $\sim 20 \%$ overestimate in the extinction (for $R_{V}=5.5, A_{K} /$ $A_{V}=0.134$; Cardelli et al. 1989). Only the molecular ridge, which comprises $<10 \%$ of the total cloud area, has $A_{V}>20$ mag.

Extinction estimates from stellar colors should be calculated using only background sources. However, the diskless members of AFGL 333 cannot be distinguished from background stars, and their inclusion in our analysis may lead to underestimating the extinction. Based on our extinctioncorrected comparison of AFGL 333 to a nearby control field (see Section 4.3), the diskless members of AFGL 333 are $\sim 30 \%$ of the background sources considered in regions with $A_{V}=10 \mathrm{mag}\left(\sim 45 \%\right.$ for $\left.A_{V}=20 \mathrm{mag}\right)$. The inclusion of diskless stars therefore moderately underestimates the extinction throughout the cloud, and is again especially severe in high extinction regions along the molecular ridge.

A smaller uncertainty is introduced by our spectral type sensitivity to background stars. The $90 \%$ completeness limit in the $K_{s}$ band is $17 \mathrm{mag}$ (see Section 2.3), which corresponds to background stars at $2 \mathrm{kpc}$ of $\mathrm{K} 7$ for $A_{V}=0 \mathrm{mag}, \mathrm{K} 4$ for 10 mag, and G4 for 20 mag. These differences will lead to an uncertainty of $\sim 2 \mathrm{mag}$ in $A_{V}$, with the ridge again most affected.

\subsection{Extinction Map from the CO Column Density}

We used the ${ }^{12} \mathrm{CO}(J=2-1)$ and ${ }^{13} \mathrm{CO} \quad(J=2-1)$ position-velocity data cubes for the entire W3 region from Bieging \& Peters (2011) to derive a gas column density map of the area around AFGL 333. The emission of these two CO isotopologues was analyzed with a non-LTE statistical equilibrium model grid and a large velocity gradient radiative transfer code (see Kulesa et al. 2005) to find the CO total column density at each map pixel. The model incorporates gas heating dominated by photons via the photoelectric effect together with the observed $\mathrm{CO}$ line intensity, to estimate the UV radiation incident at each point in the map. The $\mathrm{CO}$ abundance and total hydrogen column density $\left(N(\mathrm{H})=N(\mathrm{H} \mathrm{I})+2 N\left(\mathrm{H}_{2}\right)\right)$ are calculated following the photodissociation models of Black \& van Dishoeck (1987) and van Dishoeck \& Black (1988). The visual extinction $A_{V}$, is found using the ratio $N(\mathrm{H}) / A_{V}=1.8 \times 10^{21} \mathrm{~cm}^{-2} \mathrm{mag}^{-1}$ (Bohlin et al. 1978).

Figure 4 shows the results of this calculation, plotting the $\mathrm{CO}$-derived visual extinction $\left(A_{V}\right)$ in grayscale and the $K$-band 


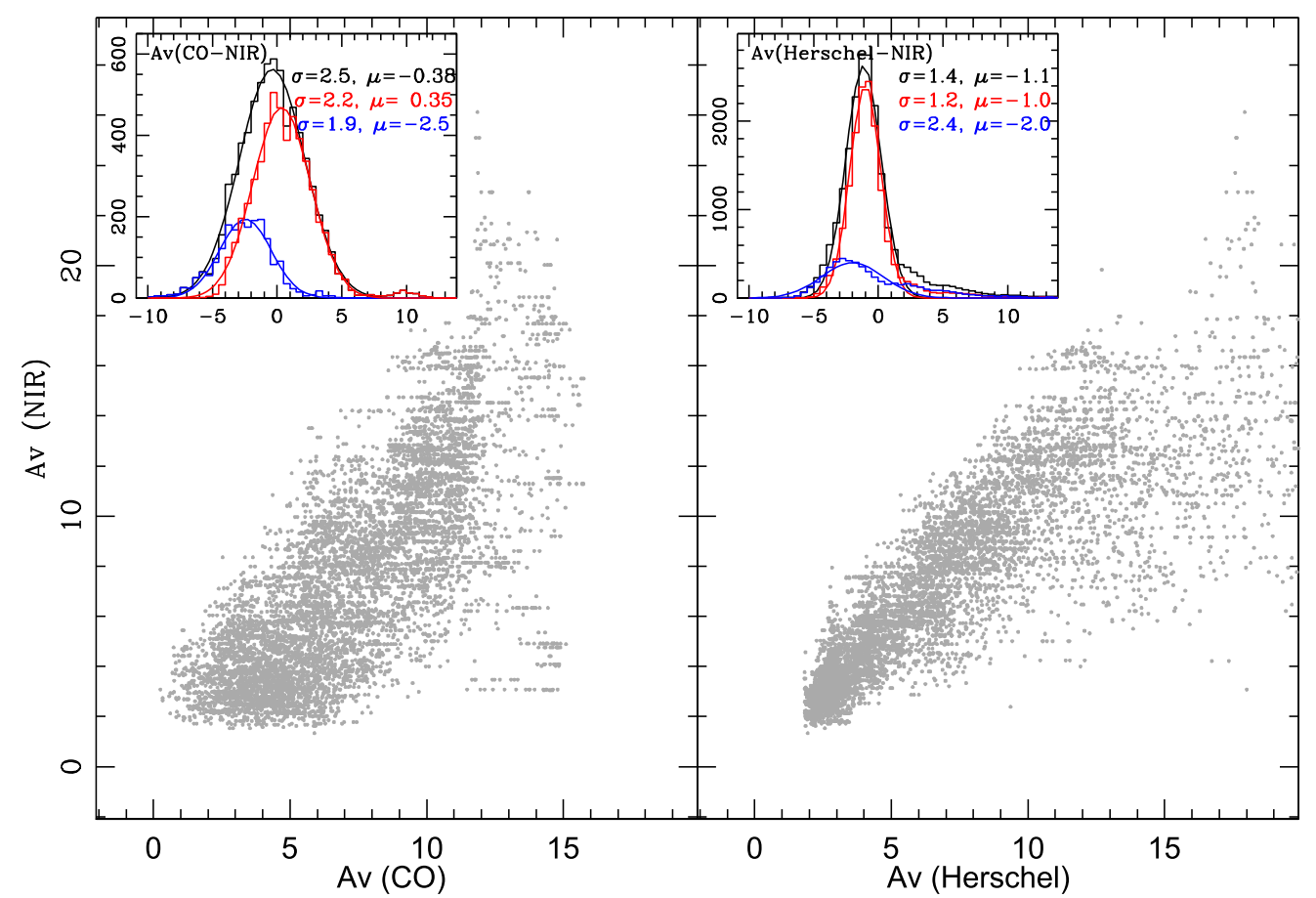

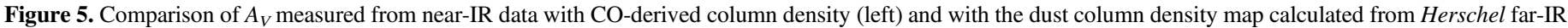

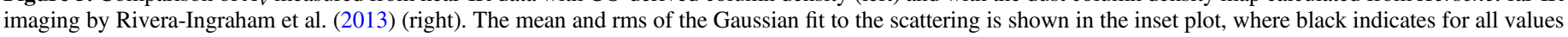
of $A_{V}$, red is for $A_{V}<10 \mathrm{mag}$, and blue is for $A_{V}>10$ mag, respectively.

extinction from the NIR point source catalog as contours. The CO-derived column density maps are subject to systematic uncertainties in the assumed values for the ${ }^{12} \mathrm{CO} /{ }^{13} \mathrm{CO}$ isotopic abundance ratio and the $\mathrm{CO} / \mathrm{H}_{2}$ molecular abundance ratio. For the galacto-centric radius of AFGL 333, we assume a ${ }^{12} \mathrm{CO} /{ }^{13} \mathrm{CO}$ isotopic ratio of 80 with a probable uncertainty of $\sim 30 \%$ (e.g., Milam et al. 2005). We assume a $\mathrm{CO} / \mathrm{H}_{2}$ abundance of $10^{-4}$ also with an uncertainty of $\sim 30 \%$. The observed $\mathrm{CO}$ lines become saturated for the highest extinction regions $\left(A_{V} \geqslant 20 \mathrm{mag}\right)$, and $\mathrm{CO}$ may become depleted onto grains at high density and low temperature. Column densities may therefore be underestimated, but this effect should be limited to a small fraction of the total area of the region.

\subsection{Comparing Extinction Maps}

Figure 4 provides qualitative support for the general cloud structure, as calculated from the near-IR extinction and CO column density. These results are also qualitatively consistent with the dust column density map calculated from Herschel farIR imaging by Rivera-Ingraham et al. (2013). However, each of these methods suffers from biases and uncertainties (e.g., Pineda et al. 2010), all of which are difficult to independently evaluate. In this subsection, we compare results from different methods to measure extinction to quantify empirical uncertainties in the extinction map.

Figure 5 compares the average near-IR extinctions to extinctions estimated from $\mathrm{CO}$ column density and Herschel dust column density maps within grids of size $38^{\prime \prime}$. Below $A_{V}=10 \mathrm{mag}$, the three sets of extinctions are well correlated. The extinctions from CO column density are $0.35 \mathrm{mag}$ in $A_{V}$ smaller than those from near-IR colors, but with a large scatter of $\mathrm{rms}=2.2 \mathrm{mag}$ between the two values. The extinctions from the far-IR dust column densities are much more tightly correlated with the near-IR extinctions, with an rms of $1.2 \mathrm{mag}$, but are $1 \mathrm{mag}$ smaller than the near-IR extinction. At $A_{V}>10 \mathrm{mag}$, these correlations become much worse. The near-IR colors overestimate high extinctions by $2 \mathrm{mag}$, with a scatter of 2-2.5 mag that is caused by a tail of very high extinction that is undetected in the near-IR.

The tight correlation between the dust emission and near-IR extinction indicates random uncertainties of $\sim 1$ mag in regions of low to modest extinction. The absolute comparisons for both the dust emission and the near-IR extinction depend sensitively on correction for foreground and background dust. The CO column density map should be robust to these uncertainties and establishes that systematic uncertainties are $\sim 0.35 \mathrm{mag}$. The near-IR extinction map becomes much more unreliable when $A_{V}>10 \mathrm{mag}$, as expected from our description of uncertainties in this method in Section 3.1. However, at high column densities, these comparisons are sensitive to the filling factor of dense gas and dust within the $38^{\prime \prime}$ boxes. If the dense material is concentrated in small regions, the near-IR extinction map may be a better estimate of the median extinction in a region. The near-IR extinction map has no sensitivity to these optically thick regions, since few background stars are detectable.

These comparisons establish that the near-IR extinction map therefore provides a reasonable estimate of the extinction to most stars in AFGL 333 at low extinction. This map will be adopted for the remainder of the paper. The extinction map and our stellar census is unreliable in the densest regions, which occur mainly along the AFGL 333 Ridge.

\section{CENSUS OF YSOS IN AFGL 333}

Several classification schemes have been developed in recent years to differentiate between YSOs with dominant envelope emission, circumstellar disk emission, and diskless sources. YSOs are often divided into Class 0, I, II, or III evolutionary stages (Lada \& Wilking 1984). Class 0 objects are deeply 
embedded protostars experiencing cloud collapse and are extremely faint at wavelengths shorter than $10 \mu \mathrm{m}$. Class I YSOs are infrared bright with emission dominated by their spherical envelopes. With only near-IR and mid-IR data it is impossible to distinguish Class 0 from Class I objects. Hereafter these objects are grouped together and called Class I in this study. A Class II YSO has no envelope and is characterized by the presence of an optically thick, primordial circumstellar disk, which gives excess emission in IR. When the circumstellar disk becomes optically thin, the star is classified as Class III.

In this section, we identify and classify Class I and Class II sources using the 1-24 $\mu \mathrm{m}$ SEDs. We do not classify the diskless Class III YSOs because they are indistinguishable from the field stars in their IR colors (see Section 5.2 for details). In the first step, we use those sources detected in all four IRAC bands to classify the objects as Class I/Class II based on their color excesses. Selection of YSOs based on the four IRAC band colors may not be complete, as we are likely to have missed many sources that fall in the region with bright nebulosity in [5.8] and [8.0] $\mu \mathrm{m}$ bands as well as due to the limited sensitivity of these two bands (see Table 1). In order to account for the missing YSOs in [5.8] and [8.0] $\mu \mathrm{m}$ bands, we identify more YSOs based on their color excess in $H, K_{s}$, [3.6], and [4.5] $\mu \mathrm{m}$. Finally, we re-examine the entire catalog of sources having $24 \mu \mathrm{m}$ photometry and more YSOs are added to the list based on the color excess of $24 \mu \mathrm{m}$ in combination with any IRAC bands. Possible contaminants are also discussed. Estimating the total population of AFGL 333 members requires identifying the diskless Class III population. Since we cannot discriminate between foreground and background stars, this estimate relies on statistical estimates of the foreground and background populations from a nearby control region. We consider a control field region from our near-IR images centered at $\alpha_{2000}=02^{\mathrm{h}} 28^{\mathrm{m}} 28^{\mathrm{s}} ; \quad \delta_{2000}=+61^{\circ} 42^{\prime} 45^{\prime \prime}, \sim 10^{\prime}$ north of BRC 5. This region is devoid of nebulosity in $K_{s}$ band. The column density map from dust emission by RiveraIngraham et al. (2013) shows that the region suffers from normal interstellar reddening. Therefore we consider this a safe region to be used as a control field region.

\subsection{Selection of YSOs Using IRAC Data}

In order to classify the YSOs detected in all IRAC bands into Class I and Class II, we adopt the color criteria given by Gutermuth et al. (2009). Of the 1567 sources detected in all the IRAC bands (see Section 2.2), 538 sources are within the AFGL 333 area considered for this study. Of these, 40 have colors consistent with Class I and 188 have colors consistent with Class II, respectively. However, in rare cases, highly reddened Class II sources could have the colors of a Class I source. In the highly reddened regions (i.e., the ridge), the average $A_{V} \sim 20 \mathrm{mag}$ can cause a shift of $\sim 0.18 \mathrm{mag}$ in the [3.6]-[4.5] color of a star (Flaherty et al. 2007). If the color excess of Class II YSOs are shifted due to the presence of reddening up to $A_{V} \sim 20 \mathrm{mag}, \sim 20 \%$ of Class I YSOs are expected to be misclassified Class II sources. In Figure 6 (left panel), the [3.6]-[4.5] versus [5.8]-[8.0] color-color diagram is shown, where the Class I and Class II sources are shown in red and green colors, respectively.

\subsection{YSOs from $H, K_{s}$, [3.6] and [4.5] $\mu \mathrm{m}$ Data}

Additional YSOs are identified based on their color excess in $H, K_{s}, 3.6$, and $4.5 \mu \mathrm{m}$ bands. We dereddened the individual point sources based on their location on the extinction map (see Figure 4) using the extinction law by Flaherty et al. (2007). We followed the various color criteria by Gutermuth et al. (2009) to classify the YSOs as either Class I or Class II. After removing the YSOs that were already identified in Section 4.1, a total of 434 more sources were added to the YSO list. Of these, 30 and 404 sources have colors consistent with Class I and Class II, respectively. However, the intrinsic uncertainty of $\sim 2 \mathrm{mag}$ in $A_{V}$ measurement (see Section 3 ) can cause a variation in the number of Class I sources by $\sim 20 \%$ and Class II sources by $\sim 7 \%$. Figure 6 (middle panel) shows the dereddened $K_{s}-$ [3.6] versus [3.6]-[4.5] color-color diagram, where Class I and Class II sources are shown in red and green colors, respectively.

\subsection{Additional YSOs from $\mathrm{H}, \mathrm{K}_{\mathrm{s}}$, and [4.5] $\mu \mathrm{m}$ Data}

We used a combination of $H, K_{s}$ and $4.5 \mu \mathrm{m}$ bands to identify the additional YSOs within AFGL 333 (Samal et al. 2014). Among all the IRAC bands, the $4.5 \mu \mathrm{m}$ band not only provides the largest number of point source detections, but also has the advantage of being unaffected by polycyclic aromatic hydrocarbons (PAHs). Three other IRAC bands are contaminated by PAHs (Whitney et al. 2008; Povich et al. 2013). Figure 7 (left panel) shows the $K_{s}-[4.5]$ versus $H-K_{s}$ color-color distribution of all sources detected within AFGL 333 along with the candidate YSOs identified in the previous sections. The reddening vector from the tip of the dwarf locus (Patten et al. 2006) is also shown.

Figure 7 (right panel) shows the color-color distribution of sources in the nearby control field region (see Section 2.4), which should have the distribution of non-YSO sources in the same Galactic direction as that of AFGL 333. A comparison of the distribution of YSOs already identified in the previous sections (Figure 7, left panel) and control field region shows that all the sources that are located toward the right side of the reddening vector are likely to have NIR excess. After excluding the YSOs that are already identified, several more sources are located toward the right side of the reddening vector. Those sources with $H-K_{s}>0.65 \mathrm{mag}$ and with an excess $>3 \sigma$ (where $\sigma$ is the average uncertainty in color) from the reddening vector (see Figure 7) are considered YSOs. Thus a total of 121 more sources are added to the YSO list. In order to confirm these sources as YSOs, we checked their location on the $H-K_{s}$ versus $K_{s}-$ [3.6] diagram and all the sources except four, which have $3.6 \mu \mathrm{m}$ detection, are found to be located toward the right side of the reddening vector. These sources also satisfy the color-color criteria to identify YSOs using $J, H$, and $4.5 \mu \mathrm{m}$ data given by Zeidler et al. (2016) (i.e., $K_{s}-[4.5]>0.49 \mathrm{mag}$ and $J-H>0.7 \mathrm{mag}$ ). Also, $\sim 70 \%$ of these sources satisfy the color and magnitude selection scheme implemented by Rivera-Ingraham et al. (2011) using $J, H, K_{s}$ data.

By selecting only those objects toward the right side of the reddening vector and by applying an additional color cut at $H-K_{s}>0.65$, we reduce the number of contaminating background objects that are reddened by the clouds. A few YSOs may be missing in this approach, but the selected sources are more reliable candidates with NIR excess (Samal et al. 

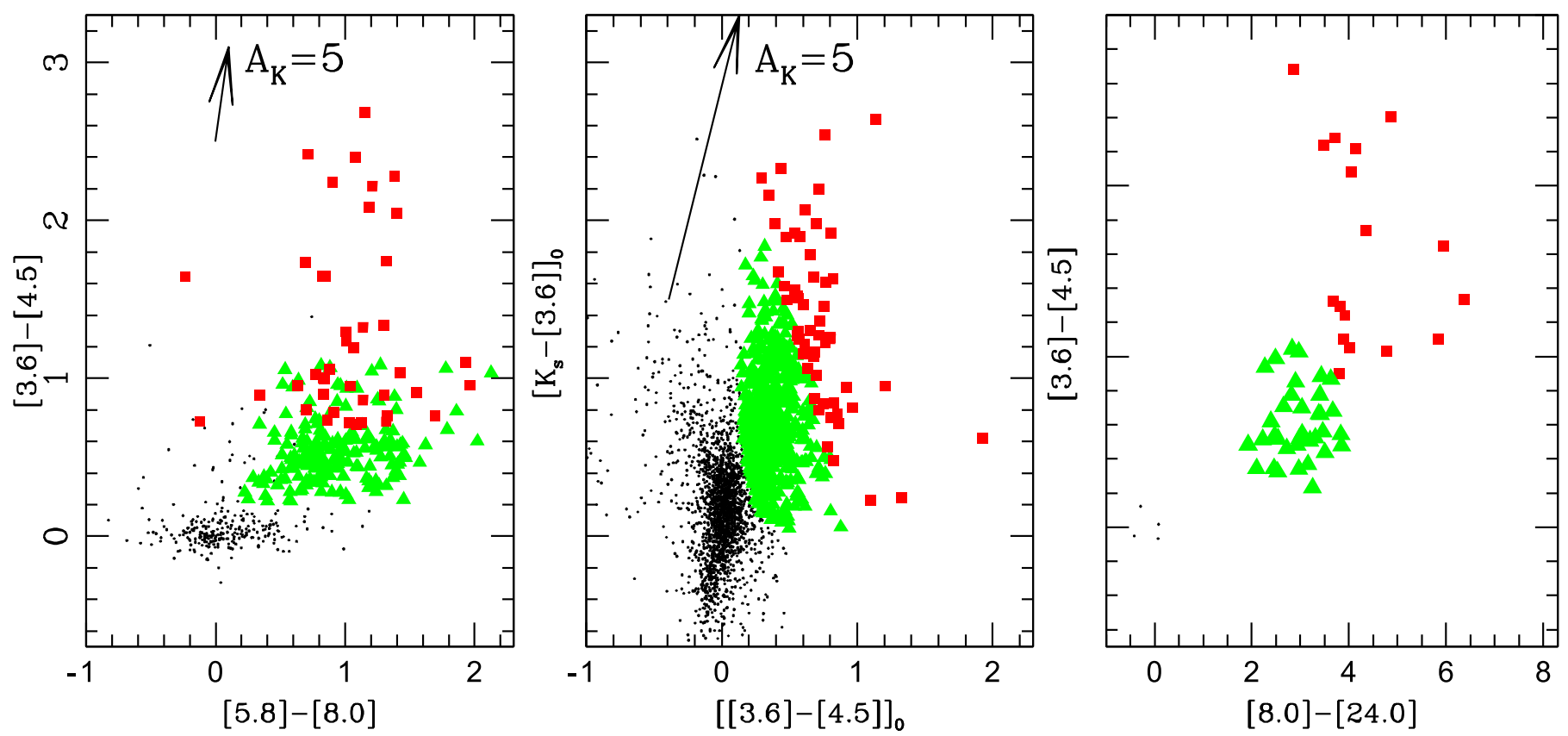

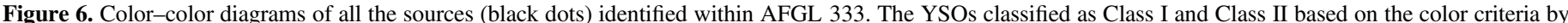

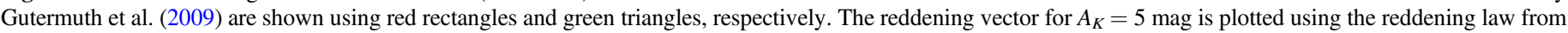

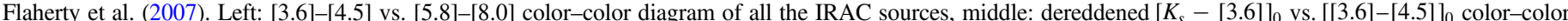

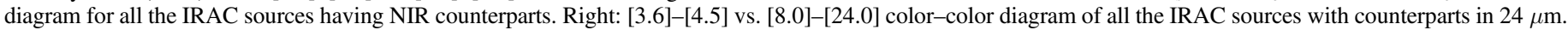
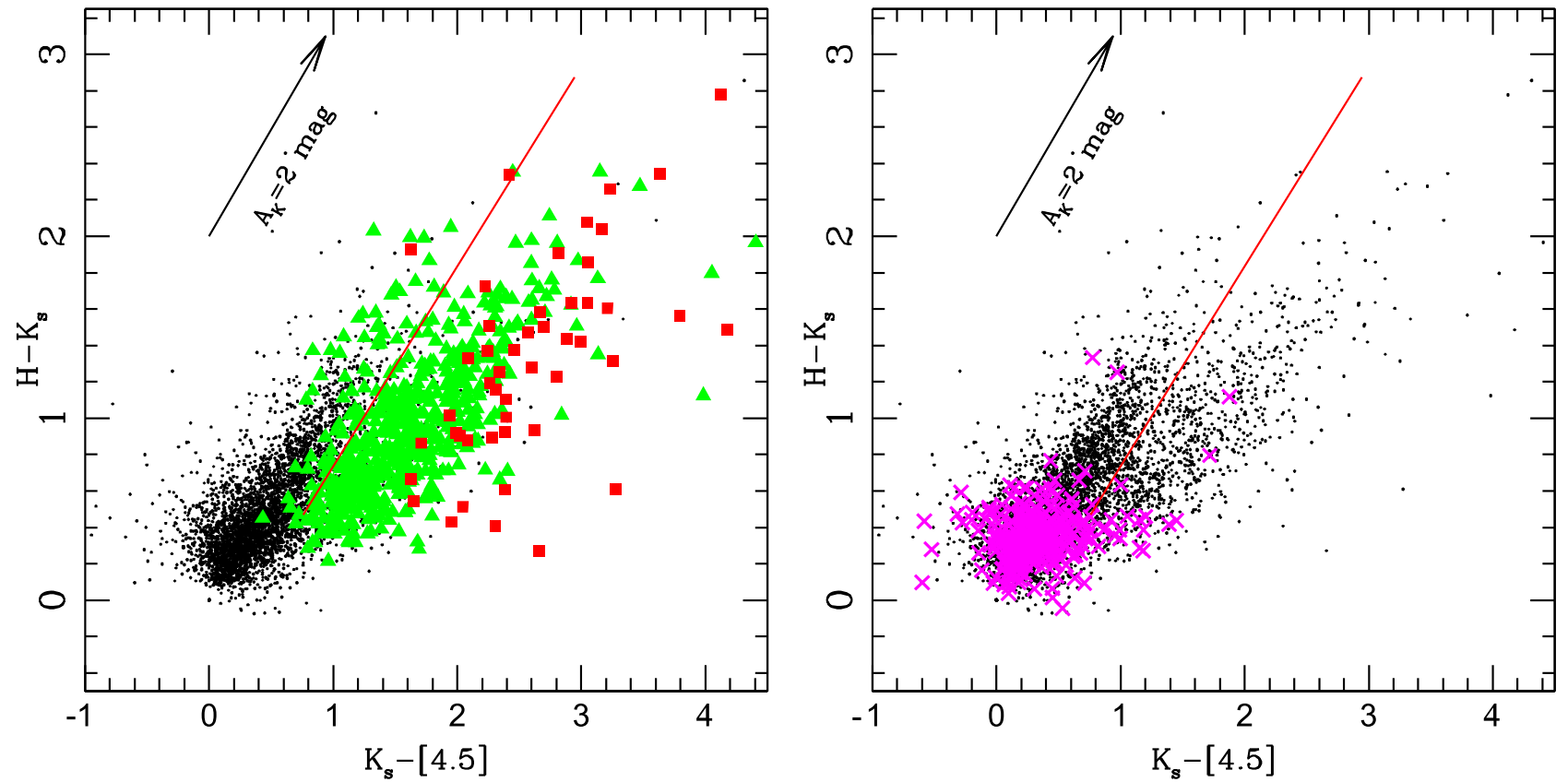

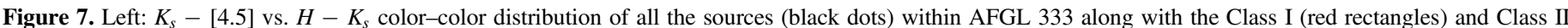

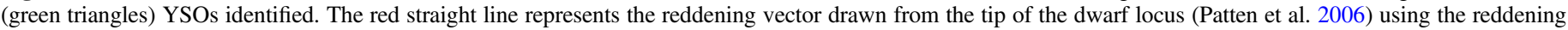
law from Flaherty et al. (2007). Right: same as in the left panel. Magenta sources represent the distribution of sources in the nearby control field.

2014; Zeidler et al. 2016). Since most of these newly identified NIR excess sources fall in the regime of the already identified Class II YSOs, we include them in the Class II category.

\subsection{YSOs Using $24 \mu \mathrm{m}$ Excess}

In the final step, the entire catalog of sources with $24 \mu \mathrm{m}$ photometry is re-examined. Any source that lacks a detection in some IRAC bands but has bright $24 \mu \mathrm{m}$ photometry (i.e., [24] $<7$ mag; [X]-[24] $>4.5$; where [X] is any IRAC band) is likely to be a deeply embedded protostar (Gutermuth et al. 2009). Using this criteria, we identify 29 new Class I sources. All previously identified Class I YSOs with MIPS detections have colors [5.8]-[24] $>4$ or [4.5]-[24] $>4$ that confirm their classification. The right panel of Figure 6 shows the [3.6]-[4.5] versus [8.0]-[24] color-color diagram of all the 
Table 2

YSO Catalog Summary in Various Bands

\begin{tabular}{lcc}
\hline \hline Band & Class I & Class II \\
\hline IRAC & 40 & 188 \\
$H, K_{s}, 3.6,4.5$ & 30 & 404 \\
{$[24]+$ IRAC(any) } & 29 & $\ldots$ \\
$H, K_{s}, 4.5$ & $\ldots$ & 121 \\
Total & 99 & 713 \\
\hline
\end{tabular}

IRAC sources having counterparts in $24 \mu \mathrm{m}$. The candidate Class I and Class II sources are marked with red squares and green triangles, respectively.

\subsection{Final Catalog of YSOs}

Our NIR and MIR color criteria using MIPS, IRAC, and NEWFIRM photometry identifies 812 candidate YSOs (99 Class Is and 713 Class IIs) associated with the AFGL 333. Table 2 summarizes the statistics of YSOs in various bands. Sample entries of photometric data of all the stars within AFGL 333 is given in Table 3, the full version of which is available in the HTML version of the journal.

The latest census of YSOs (Class I and Class II) in AFGL 333 by Rivera-Ingraham et al. (2011) identified 156 YSOs within the area considered in this study. Of these, 138 are recovered in our YSO list (37 Class I, 101 Class II). The slight discrepancy in the number of YSOs is due to the different YSO selection criteria adopted in these two studies. Of the 37 Class I sources in our list, 31 are classified as Class 0/I and 6 as Class II in Rivera-Ingraham et al. (2011). Of the 101 Class II sources in our list, 91 are classified as Class II and 10 as class I in their list. They have also identified an additional 83 candidate premain-sequence sources in the region using a 2MASS-based color-color and magnitude selection scheme. Of these, 70 are recovered in our YSO list (3 Class I, 67 Class II). Their survey was limited by the sensitivity of 2MASS data. In conclusion, the previous survey of this region identified 239 candidate YSOs and due to the improved sensitivity of $J H K_{s}$ data in this study by $>3$ mag in each bands, we have identified 573 more YSOs within AFGL 333.

\subsection{Source Contamination Rate}

Our candidate YSO population may be contaminated by the background sources including PAH-emitting galaxies, broadline active galactic nuclei (AGNs), unresolved knots of shock emission, PAH-emission-contaminated apertures, etc. that mimic the colors of YSOs. Since we are observing through the Galactic plane, contamination due to galaxies should be negligible (Massi et al. 2015). In order to have a statistical estimate of possible galaxy contamination in our YSO sample, we used the Spitzer Wide-area Infrared Extragalactic (SWIRE) catalog coming from the observations of the ELAIS N1 field (Rowan-Robinson et al. 2013). SWIRE is a survey of the extragalactic field using Spitzer IRAC and MIPS bands and can be used to predict the number of galaxies with colors that overlap with YSO colors (Evans et al. 2009). The SWIRE catalog is resampled for the spatial extent as well as the sensitivity limits of our observations in AFGL 333 and is also reddened by the average reddening of AFGL 333 (i.e., $A_{V}=10$ mag, see Section 3). The YSO selection criteria is applied to the resampled SWIRE catalog and only $\sim 2 \%$ of our YSO sample is found to be compatible with the galaxy colors. Similarly, using the color criteria given by Robitaille et al. (2008), $\sim 2 \%$ of YSOs seem to have colors consistent with AGB stars.

Finally, a comparison between the distribution of control field stars and the YSOs (see Figure 7) shows that $<5 \%$ of YSOs coincide with the location of field stars. In summary, the contribution of various contaminants in our YSO sample is $<5 \%$ (i.e., galaxies $\sim 2 \%$, AGBs $\sim 2 \%$ ), which is a small fraction of the total number of YSOs.

We also applied the Gutermuth et al. (2009) color-color criteria to the candidate YSOs identified within AFGL 333. In Table 2, $\sim 38 \%$ of Class I and $\sim 15 \%$ of Class II of the final YSO list are matched with the colors of PAH contaminated apertures, shock emissions, PAH-emitting galaxies, and AGNs. The major contribution is from the contamination by AGNs. However, several studies (e.g., Koenig et al. 2008; RiveraIngraham et al. 2011; Willis et al. 2013) have noticed that the use of Gutermuth et al. (2009) criteria for a region at $\sim 2 \mathrm{kpc}$ would likely provide an overestimation of the contamination.

\subsection{Mass Completeness Limit of YSOS}

The $J$ versus $(J-H)$ and $H$ versus $\left(H-K_{s}\right)$ colormagnitude diagrams (Figure 8) are used to get an estimate of the mass range of the candidate YSOs identified within AFGL 333. The Class I and Class II YSOs identified from various color combinations of IRAC and NIR bands in Section 4 are shown using red rectangles and green triangles, respectively, in Figure 8. The pre-main-sequence isochrone with age 2 Myr (Bressan et al. 2012) and reddening vectors for several masses are also shown. The wide variation in the colors of YSOs in Figure 8 is caused by variable extinction and different evolutionary stages of sources within AFGL 333. In general, the majority of YSOs lie in the mass range $\sim 0.1-2 M_{\odot}$.

The mass completeness limit for our YSO survey is dictated by the photometric completeness and wide range of YSO colors. We selected the YSO sample based on the IRAC, $H K_{s}+$ IRAC, and IRAC+MIPS color combinations (see Section 4). Within AFGL 333, 85\% of the Class I sources have counterparts in IRAC $4.5 \mu \mathrm{m}$ band and all the Class II sources are detected in $4.5 \mu \mathrm{m}$ (see Table 2). The $90 \%$ photometry completeness limits of $K$ and $4.5 \mu \mathrm{m}$ bands are estimated to be $\sim 17$ and 16 mag, respectively (see Section 2.3). Assuming a distance of $2.0 \mathrm{kpc}$ for AFGL 333 and an average extinction $A_{V}$ of $\sim 10 \mathrm{mag}$ (see Section 3 ), the photometric completeness limits in these bands correspond to an approximate stellar mass of $\sim 0.2 M_{\odot}$ for a YSO of age $\sim 2 \mathrm{Myr}$ (using the evolutionary tracks of Bressan et al. 2012). The completeness limit is $>0.4 M_{\odot}$ at high extinction regions such as the AFGL 333 Ridge, where the extinction $A_{V}>20$ mag. However, only $<10 \%$ of the total area suffers this high extinction. Also, due to the IR excess, the YSOs will be brighter in IR bands compared to their main-sequence counterparts with only photospheric emission. So the completeness limits of Class I and Class II sources will be lower than $0.2 M_{\odot}$, when compared to the diskless sources. The previous YSO survey of this region (Rivera-Ingraham et al. 2011) was limited by the 2MASS completeness limit, which corresponds to $>1 M_{\odot}$ for $A_{V} \sim 10$ mag. With the deep photometry, the current analysis probes the very low mass stars $\left(>0.2 M_{\odot}\right)$ of the region. For further analysis, we include only those sources brighter than 16 mag in $4.5 \mu \mathrm{m}$. 
Table 3

Photometric Data of All Point Sources within AFGL 333

\begin{tabular}{|c|c|c|c|c|c|c|c|c|c|c|}
\hline $\begin{array}{l}\alpha_{2000} \\
(\mathrm{deg})\end{array}$ & $\begin{array}{l}\delta_{2000} \\
(\mathrm{deg})\end{array}$ & $\begin{array}{c}J \\
(\mathrm{mag})\end{array}$ & $\begin{array}{c}H \\
(\mathrm{mag})\end{array}$ & $\begin{array}{c}K_{s} \\
(\mathrm{mag})\end{array}$ & $\begin{array}{l}{[3.6]} \\
\text { (mag) }\end{array}$ & $\begin{array}{l}{[4.5]} \\
(\mathrm{mag})\end{array}$ & $\begin{array}{l}{[5.8]} \\
(\mathrm{mag})\end{array}$ & $\begin{array}{c}{[8.0]} \\
(\mathrm{mag})\end{array}$ & $\begin{array}{l}\text { [24] } \\
\text { (mag) }\end{array}$ & Class \\
\hline 36.9357 & +61.6750 & 12.10 & 11.41 & 10.88 & 9.98 & 9.74 & 9.38 & 9.13 & $\ldots$ & Class II \\
\hline 36.6905 & +61.6978 & 12.16 & 11.49 & 10.93 & 9.53 & 9.19 & 8.40 & 7.64 & 5.18 & Class II \\
\hline 36.6395 & +61.6879 & 12.68 & 11.96 & 11.53 & 11.13 & 10.74 & $\ldots$ & ... & $\ldots$ & Class II \\
\hline 37.1809 & +61.5262 & 12.81 & 12.28 & 11.88 & 11.21 & 10.90 & 10.72 & 10.53 & $\ldots$ & Class II \\
\hline 36.7891 & +61.9122 & 12.87 & 12.14 & 11.72 & 11.23 & 10.96 & 10.67 & 10.31 & $\ldots$ & Class II \\
\hline
\end{tabular}

(This table is available in its entirety in machine-readable form.)
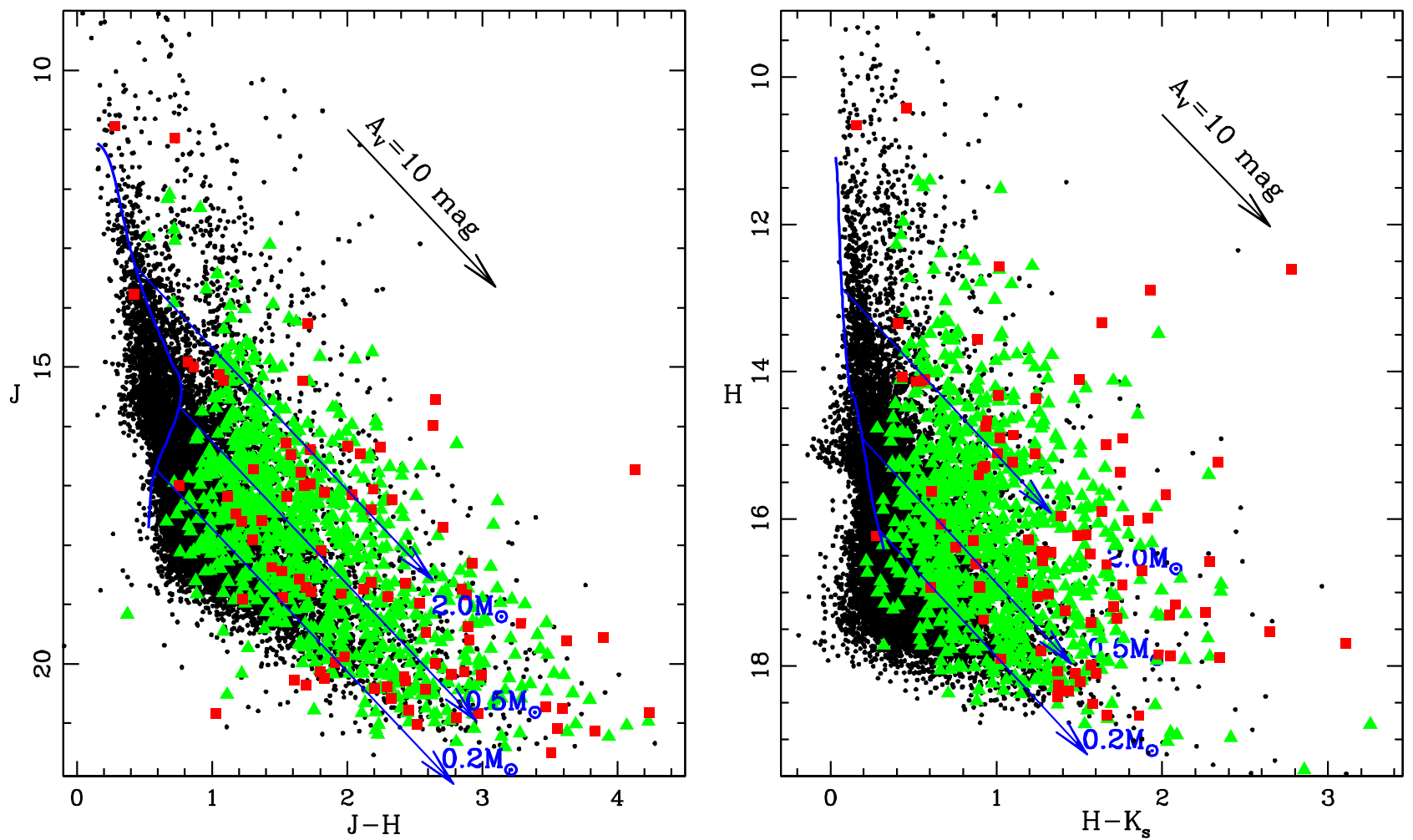

Figure 8. $J$ vs. $(J-H)$ (left) and $H$ vs. $\left(H-K_{s}\right)$ (right) color-magnitude diagrams of all the sources (black dots) within the AFGL 333 region. The red rectangles and green triangles are the Class I and Class II YSOs identified in Section 4. The blue solid curve is the pre-main-sequence isochrone of age $2 \mathrm{Myr}$ (Bressan et al. 2012) shifted for a distance of $2.0 \mathrm{kpc}$. The straight lines represent the reddening vectors for $A_{V}=20 \mathrm{mag}$ that correspond to stellar masses of $0.2,0.5$, and $2 M_{\odot}$.

\section{CHARACTERISTICS OF YSOS, ENVIRONMENT, AND STAR FORMATION IN AFGL 333}

In this section the final YSO catalog obtained from Section 4 is used to obtain their spatial distribution, mass distribution, stellar surface density, and clustering properties.

\subsection{Spatial Distribution of YSOs and Sub-clustering in AFGL 333}

The spatial distribution of YSOs can probe the fragmentation processes that lead to the formation of protostellar cores and the subsequent dynamical evolution of star-forming regions. Figure 9 shows the spatial distribution of the candidate YSOs (i.e., red: Class I; green: Class II;) overlaid on the $4.5 \mu \mathrm{m}$ Spitzer image, where the YSOs are preferentially located in/ around the regions of high extinction (see Figure 4). The Class I YSOs follow the elongated nature of the molecular ridge seen in the CO maps of Sakai et al. (2006) and Bieging \& Peters (2011) and in our extinction map shown in Figure 4.

Since W3 is a complex star-forming region, a high level of sub-clustering is expected and is evident in the spatial distribution of candidate YSOs (Figure 9, left panel). In order to understand the level of sub-clustering within AFGL 333, we generated the stellar density map using the nearest neighborhood technique (Gutermuth et al. 2005). We followed the method introduced by Casertano \& Hut (1985), where the stellar density $\sigma(i, j)$ inside a cell of a uniform grid with its center at the coordinates $(i, j)$ is

$$
\sigma(i, j)=\frac{N-1}{\pi r_{N}^{2}(i, j)}
$$

where, $r_{N}$ is the distance from the center of the cell to the $N$ th nearest source. The value of $N$ is allowed to vary depending on 

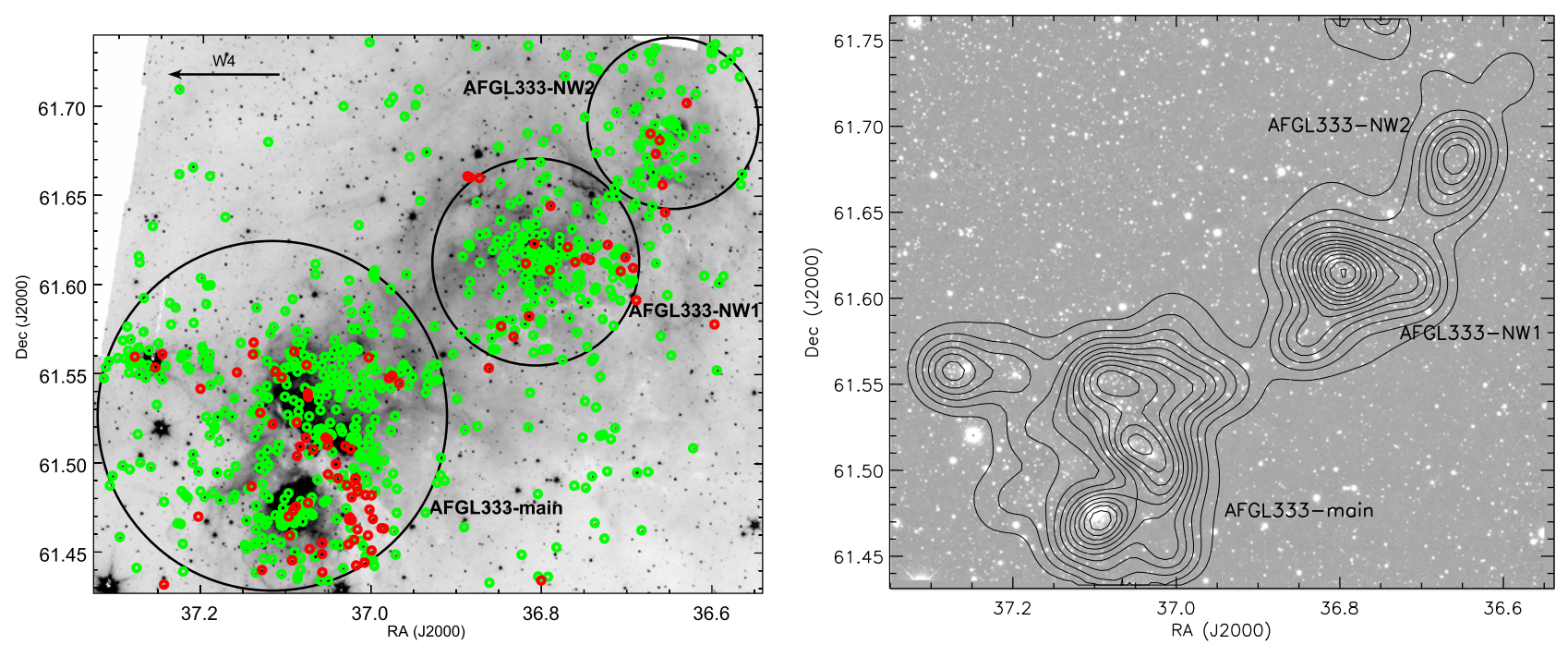

Figure 9. Left: spatial distribution of candidate YSOs identified within AFGL 333 overlayed on the $4.5 \mu \mathrm{m}$ Spitzer-IRAC image. Class I, Class II YSOs are represented in red and green, respectively. The important stellar groups identified within AFGL 333 are labeled and the circles around them represent their estimated sizes. Right: surface density map of YSOs within AFGL 333 overlayed on the NEWFIRM $K_{s}$-band image. Surface density was calculated using the six nearest neighbors. Contours correspond to YSO surface densities of 5-44 stars $\mathrm{pc}^{-2}$ with an interval of $3 \mathrm{stars} \mathrm{pc}^{-2}$.

the smallest scale structures of the regions of interest. The surface density map for the candidate YSOs detected above the completeness limit is generated with a grid size of $10^{\prime \prime} \times 10^{\prime \prime}$ and $N=6$ as a compromise between resolution and sensitivity of the map.

In the surface density map shown in the right panel of Figure 9, three major groups of YSOs are identified, which agrees with our visual interpretation of the spatial distribution of YSOs. The main over-density corresponds to the group of YSOs associated with the AFGL 333 Ridge and its surroundings, which itself appears to have multiple density peaks. Hereafter we call this group of YSOs AFGL 333 Main. Within AFGL 333 Main, the YSO surface density peaks at the center of the cluster associated with the $\mathrm{H}$ II region (i.e., IRAS 02245 +6115 ; see Figure 2). The second over-density of YSOs is located toward the north of the AFGL 333 Ridge, $\left(\alpha_{2000}=02^{\mathrm{h}} 27^{\mathrm{m}} 14^{\mathrm{s}} ; \delta_{2000}=+61^{\circ} 36^{\prime} 48^{\prime \prime}\right)$ and the YSOs are associated with a mid-IR cavity seen in the background image. The majority of YSOs in this cluster are Class II sources. Hereafter we call this group of YSOs AFGL 333-NW1. One more cluster (AFG 333-NW2) is seen further northwest of the region $\left(\alpha_{2000}=02^{\mathrm{h}} 26^{\mathrm{m}} 34^{\mathrm{s}} ; \quad \delta_{2000}=+61^{\circ} 41^{\prime} 27^{\prime \prime}\right)$ and $\sim 70$ YSOs are associated with it.

Some of these groups of YSOs are part of known clusters in the literature. For example, the sub-group associated with BRC 5 , located at the outermost boundary of the HDL (IRAS 02252 +6120 ) and with the $\mathrm{H}_{\text {II }}$ region (IRAS 02245+6115), host clusters (Bica et al. 2003). The latest clustering analysis of the W3 complex using the minimum spanning tree method by Rivera-Ingraham et al. (2011) identified eight stellar groups (IDs 0 to 7 ) as part of AFGL 333. Of these, five groups (IDs 1, $3,4,6$, and 7) are within the area considered in this study and others are outside the area. Groups 6 and 4 are associated with AFGL 333-NW1 and groups 1, 3, and 7 belong to AFGL 333 Main. The individual stellar peaks in Figure 9 coincide with that of the stellar groups identified by Rivera-Ingraham et al. (2011). Though the individual groups are assumed to be at various evolutionary stages and at different environments (see
Rivera-Ingraham et al. 2011 for details) due to our limited spatial resolution and detection sensitivity in this study we are unable to segregate the various sub-groups within AFGL 333 Main and hence tentatively consider them a single group of YSOs.

Based on the YSO density distribution, the extent of individual groups within AFGL 333 is estimated from the radius of the outermost stellar density contour (see Figure 9) around each cluster, within which $\sim 90 \%$ of the candidate YSOs are concentrated. Thus radii of $6 ! .0(3.5 \mathrm{pc}), 3 ! 5(2.0 \mathrm{pc})$, and $3 ! .0(1.7 \mathrm{pc})$ are considered for AFGL 333 Main, AFGL 333 NW1, and AFGL 333 NW2, respectively. Radii of the individual groups are marked in Figure 9. For AFGL 333 NW1 and AFGL 333 NW2, stellar density peaks are considered as their center, whereas AFGL 333 Main has multiple density peaks and hence the center of the circle that covers the outermost density contour is tentatively considered its center.

\subsection{Properties of Stellar Groups in AFGL 333: Disk Fraction and Ages}

In this subsection we discuss the disk fraction and ages of three stellar groups identified within AFGL 333. Though these individual groups have sub-clusters in them (Figure 9), due to the limited sensitivity and spatial resolution of the present study, we consider them a single group of YSOs. Hence the properties of the individual groups studied below should be considered their average properties. The statistics of various sources within individual groups as well as in a nearby control field region are summarized in Table 4.

An approximate age estimation of individual groups is difficult to determine from the color-magnitude diagram because the luminosity scatter is large due to NIR excess and extinction (e.g., Figure 8). An alternate way to roughly infer the ages of young clusters is from the disk fraction since the fraction of sources surrounded by circumstellar disks strongly depends on the age of the system (Haisch et al. 2001; Hernández et al. 2008; Fang et al. 2013). From Spitzer IRAC 
Table 4

Derived Cluster Parameters

\begin{tabular}{|c|c|c|c|c|c|c|}
\hline $\begin{array}{l}\text { Group } \\
\text { ID }\end{array}$ & Radius & $\begin{array}{l}\text { Total } \\
\text { Stars }\end{array}$ & Class I & Class II & $N(\mathrm{I} / \mathrm{II})$ & $\begin{array}{l}\text { Disk Fraction } \\
\left(A_{V} \text { Corrected }\right)\end{array}$ \\
\hline AFGL 333 Main & $6{ }^{\prime} 0(3.5 \mathrm{pc})$ & 1647 & 58 & 372 & 0.16 & $61 \%$ \\
\hline AFGL 333-NW1 & $3.5(2.0 \mathrm{pc})$ & 615 & 14 & 158 & 0.09 & $58 \%$ \\
\hline AFGL 333-NW2 & $3 ! 0(1.7 \mathrm{pc})$ & 378 & 5 & 66 & 0.08 & $50 \%$ \\
\hline Control field & $3 ! 0(1.7 \mathrm{pc})$ & 279 & $\cdots$ & $\ldots$ & $\ldots$ & $\ldots$ \\
\hline
\end{tabular}

studies of young clusters, the average disk fractions at different cluster ages are $75 \%$ at $\sim 1 \mathrm{Myr}, 50 \%$ at $\sim 2 \mathrm{Myr}, 20 \%$ at $\sim 5 \mathrm{Myr}$, and 5\% at $10 \mathrm{Myr}$ (Williams \& Cieza 2011).

The census of the diskless Class III sources is unknown in this survey because of their lack of IR excess, and because Xray or spectroscopic data are not available to identify them. In order to account for the total number of Class III sources, the statistics of the point sources in a nearby control field is used (Table 4). We subtracted the field star contribution from the individual groups after scaling the number of sources in the control field to the areas of individual groups. The remaining number of sources is considered to be the total number of member stars (disk and diskless) within each region. However, a direct subtraction of field stars would overestimate the disk fraction because the background sources within the sub-regions are seen through the intervening molecular cloud, whereas the control field stars are only affected by the normal interstellar reddening. To account for differential extinction between AFGL 333 and our control field, we synthesized the background stellar population using the Besançon Galactic model of stellar population (Robin et al. 2003) for normal interstellar reddening and for $A_{V}=10 \mathrm{mag}$. Here we assume $A_{V}=10 \mathrm{mag}$ as the average reddening within AFGL 333 (see Section 3). We find that $\sim 85 \%$ of field stars will be seen through the cloud in the $L$-band when compared to the total number of field stars in a cloud-free region.

Table 4 provides the disk fraction of individual clusters. After accounting for extinction, the disk fraction is estimated to be $\sim 61 \%, 58 \%$, and $50 \%$ for AFGL 333 Main, AFGL 333 NW1, and AFGL 333 NW2, respectively. The average disk fraction versus age trend reported in the literature (e.g., Haisch et al. 2001; Hernández et al. 2008; Fang et al. 2012, 2013) suggests $\sim 50 \%$ of the stars in a given region should have lost their disks around 2-3 Myr. The disk fraction estimated for AFGL 333 matches with star-forming regions of age 2-3 Myr.

Another way to infer the relative ages of different cluster populations is from the Class I/II ratio, given the different median lifetimes for these two evolutionary phases (i.e., $0.44 \mathrm{Myr}$ for Class I and $2 \mathrm{Myr}$ for Class II; Evans et al. 2009). The ratio of Class I to Class II sources should decrease with the average age of the region (Beerer et al. 2010; Myers 2012). Within the sub-clusters of AFGL 333, the Class I/II ratios are $\sim 10 \%$ (see Table 4 ), indicating a low fraction of embedded stellar population. The Class I/II value for AFGL 333 is comparable to the Vul OB1 (Billot et al. 2010) and Cygnus X north (Beerer et al. 2010), which are at similar distances to AFGL 333. After scaling to the distance of AFGL 333, the Class I/II ratio of some of the c2d clouds (Evans et al. 2009) and young clusters (Gutermuth et al. 2009) agrees with that of AFGL 333 Main (Kim et al. 2015). Assuming the same distance, all of the above regions and AFGL 333 have similar Class I/II values, which indicates that they are similar in age ( $\sim 2-3 \mathrm{Myr})$ or at a similar evolutionary stage.

As evident in Figure 9, the number of Class I sources increases toward the center of the ridge. The Class I/II ratio $\left(\sim 1.3\right.$; 32 Class I, 24 Class II) within $2 \mathrm{pc}^{2}$ of the ridge is high when compared to $\sim 0.08$ in the rest of the cloud. Therefore the ridge is likely young ( $<1 \mathrm{Myr}$, Evans et al. 2009; Samal et al. 2015), while other regions within AFGL 333 are older. This is in agreement with Rivera-Ingraham et al. (2011) who found that the stellar group associated with the molecular ridge is relatively younger than that of other stellar groups.

The above methods to estimate the age of the region should be taken with caution. The disk fraction can only be used as a rough age indicator because of large scatter in this relation due to several factors including the incompleteness of different surveys, differences in diagnostic methods used for disk fraction estimation, and the effects that age spread, metallicity, UV radiation, and stellar density may have on the disk evolution (Soderblom et al. 2014; Spezzi et al. 2015). Similarly, the Class I/II ratio is sensitive to the age spreads and also due to the uncertainty in the Class 0/I lifetimes. Moreover, the disk fraction of Class I/II ratios of AFGL 333 are the average values of the individual groups, which contains several sub-groups and are probably at various evolutionary stages.

\subsection{Estimation of Cloud Mass}

In Section 3, we constructed an extinction map of resolution $20^{\prime \prime}$ for the AFGL 333 region using the deep NIR data. From the extinction map and assuming a distance of $2.0 \mathrm{kpc}$, the cloud mass of AFGL 333 is estimated using the following relation $M=m_{\mathrm{H}} \mu_{\mathrm{H}_{2}} A \Sigma N\left(\mathrm{H}_{2}\right)$, where $m_{\mathrm{H}}$ is the mass of hydrogen, $\mu_{\mathrm{H}_{2}}$ is the mean molecular weight per $\mathrm{H}_{2}$ molecule, $N\left(\mathrm{H}_{2}\right)$ is the column density, and $A$ is the pixel area. The value of $\mu_{\mathrm{H}_{2}}$ used is 2.8 (Kauffmann et al. 2008). We convert the extinction in magnitude to column density using the relation $N$ (H) $/ A_{V}=1.87 \times 10^{21} \mathrm{~cm}^{-2} \mathrm{mag}^{-1}$ (Bohlin et al. 1978). This expression has been derived assuming $R_{V}=3.1$. After subtracting the foreground reddening, the column density of all pixels within AFGL 333 is integrated to estimate the cloud mass. The estimated mass within the AFGL 333 area is $\sim 1.7 \times 10^{4} M_{\odot} \quad$ which is consistent with the mass $\left(\sim 1.8 \times 10^{4} M_{\odot}\right)$ estimated from the CO-based column density map. We explain below the various uncertainties involved in the mass estimation from the NIR-based extinction map and infer a corrected mass.

The major uncertainty in the estimation of cloud mass comes from the assumption of a standard extinction law in the complex. Use of $R_{V}=3.1$ would overestimate the cloud mass by a factor of 1.4 when compared to $R_{V}=5.5$ (i.e., $N_{\mathrm{H}} /$ $A_{V}=1.37 \times 10^{21} \mathrm{~cm}^{-2} \mathrm{mag}^{-1}$, Draine 2003; Evans et al. 2009; Heiderman et al. 2010). Similarly, there is an intrinsic uncertainty up to $\sim 2$ mag in every pixel of the $A_{V}$ map (see 
Table 5

Cluster Parameters

\begin{tabular}{|c|c|c|c|c|c|c|c|}
\hline $\begin{array}{l}\text { Group } \\
\text { ID }\end{array}$ & $\begin{array}{l}\text { Area } \\
\left(\mathrm{pc}^{2}\right)\end{array}$ & $\begin{array}{c}M_{\text {cloud }}{ }^{\mathrm{a}} \\
\left(M_{\odot}\right)\end{array}$ & $\begin{array}{l}M_{\text {star }} \\
\left(M_{\odot}\right)\end{array}$ & $\overline{\mathrm{SFE}}$ & $\begin{array}{c}\mathrm{SFR}^{\mathrm{b}} \\
\left(M_{\odot} \mathrm{Myr}^{-1}\right)\end{array}$ & $\begin{array}{c}\Sigma_{\mathrm{gas}} \\
\left(M_{\odot} \mathrm{pc}^{-2}\right)\end{array}$ & $\begin{array}{c}\Sigma_{\mathrm{SFR}} \\
\left(M_{\odot} \mathrm{Myr}^{-1} \mathrm{pc}^{-2}\right)\end{array}$ \\
\hline AFGL 333 Main & 38.0 & $7100-7700$ & 248 & $0.03-0.03$ & $78-118$ & $187-203$ & $2.1-3.1$ \\
\hline AFGL 333-NW1 & 12.8 & $940-1150$ & 123 & $0.10-0.12$ & $30-45$ & $73-90$ & $2.3-3.5$ \\
\hline AFGL 333-NW2 & 9.5 & $660-810$ & 69 & $0.08-0.10$ & $20-31$ & $69-85$ & $2.2-3.3$ \\
\hline Total & 60.3 & $8700-9700$ & 440 & $0.04-0.05$ & $128-192$ & $144-161$ & $2.1-3.2$ \\
\hline
\end{tabular}

Notes.

a The cloud mass is estimated after incorporating the uncertainty in extinction measurement.

b SFR is calculated for ages 2 and 3 Myr.

Section 3). Also, the estimated cloud mass should be considered as a lower limit since the measured column density from the extinction map is limited by the detection limit of our NIR observations (i.e., $A_{V}<26 \mathrm{mag}$ ), especially toward the ridge where the column density is high.

A small fraction of the total area of AFGL 333 (i.e., 4\%), close to the center of the ridge, has an average column density of $\sim 3.7 \times 10^{22} \mathrm{~cm}^{-2}$ (Higuchi et al. 2013). The mass of the molecular clump around the ridge was measured as $\sim 4.2 \times 10^{3} M_{\odot}$ by Higuchi et al. (2013) using $\mathrm{C}^{18} \mathrm{O}(J=1$ $-0)$ line emission. Including the extra cloud mass, which is underestimated in our extinction map, the total mass of the AFGL 333 region is estimated to be $\sim 2.1 \times 10^{4} M_{\odot}$. The intrinsic uncertainty of $\sim 2 \mathrm{mag}$ in the $A_{V}$ measurement (see Section 3) is added to the extinction map in order to estimate the upper limit of the cloud mass, which is obtained as $\sim 2.6 \times 10^{4} M_{\odot}$. The cloud mass estimate of AFGL 333 per area is consistent with that of Rivera-Ingraham et al. (2013). AFGL 333 region contains $\sim 10 \%$ of the total mass of the entire W3 complex measured from ${ }^{12} \mathrm{CO}(J=3-2),{ }^{13} \mathrm{CO}(J=3-2)$ and $\mathrm{C}^{18} \mathrm{O}(J=3-2)$ maps $\left(\sim 4.4 \times 10^{5} M_{\odot}\right.$; Polychroni et al. 2012) and from the Herschel far-IR dust emission map $\left(\sim 2.3 \times 10^{5} M_{\odot}\right.$; Rivera-Ingraham et al. 2013).

We also calculated the cloud masses around individual stellar groups by integrating the cloud density within their respective area, after accounting for the cloud mass toward the ridge and the intrinsic uncertainty in extinction measurements (see Table 5).

\subsection{Star Formation Efficiency and Rate}

Star formation efficiency (SFE) and rate are two fundamental physical parameters to characterize how the cloud mass is converted into stars. We derive the star formation efficiency of the region by comparing the mass of the gas reservoir $\left(M_{\text {cloud }}\right)$, with the mass that has turned into stars $\left(M_{\text {star }}\right)$ during the last few million years, i.e., $\mathrm{SFE}=M_{\text {star }} /\left(M_{\text {star }}+M_{\text {cloud }}\right)$ (Myers et al. 1986).

In order to estimate the stellar mass, the total number of stars detected within individual stellar groups and brighter than the completeness limit $\left(K_{s}<17 \mathrm{mag}\right.$, for $>0.2 M_{\odot}$ at $A_{V}<10$ mag) is used. We subtracted the extinction-corrected field star contribution from individual groups (see Section 5.2). The remaining stars are normalized to the Kroupa IMF with $\alpha=2.3$ for $M>0.5 M_{\odot}$ and $\alpha=1.3$ for $M<0.5 M_{\odot}$ (Kroupa 2002). The total stellar mass is derived by extrapolating down to the hydrogen-burning limit $\left(0.08 M_{\odot}\right)$. The estimated stellar mass within each stellar group and the total stellar mass of all three groups are given in Table 5.
The overall star formation efficiency for various stellar groups ranges between $\sim 3 \%$ and $11 \%$. Their combined star formation efficiency is $\sim 4.5 \%$. In general, the star formation efficiency of AFGL 333 is consistent with that of the majority of the nearby star-forming regions in the Galaxy (i.e., 3\%-6\%, Evans et al. 2009).

SFR is the rate at which the gas in a cloud turns into stars. $\mathrm{SFR}$ can be estimated using the relation $\mathrm{SFR}=M_{\text {cloud }} \times \mathrm{SFE} /$ $t_{\mathrm{SF}}$, where $t_{\mathrm{SF}}$ is the duration of star formation. The SFRs of the individual stellar groups as well as for the entire region are estimated by assuming the duration of star formation in AFGL 333 is $\sim 2-3 \mathrm{Myr}$ (see Table 5). On average, the AFGL 333 region is forming $\sim 130-190 M_{\odot}$ of stars every Myr. The SFR varies with gas density $\left(\Sigma_{\text {gas }}\right)$, i.e., the lowdensity regions have SFRs less than the high-density regions within AFGL 333 (see Table 5). This result agrees with the analysis of nearby molecular clouds (Lada et al. 2010; Gutermuth et al. 2011), Spitzer c2d/GB clouds (Heiderman et al. 2010), and galactic massive dense clumps (Wu et al. 2010) where they find a linear relation between SFR and local gas density.

The SFR per unit surface area $\left(\Sigma_{\mathrm{SFR}}\right)$, i.e., the density of star formation, has been considered as a more generalized representation of the SFR of a given region (Heiderman et al. 2010). $\Sigma_{\text {SFR }}$ for individual groups within AFGL 333 has been estimated by considering the area of each cluster over which the cloud mass is measured (see Table 5). The derived values of $\Sigma_{\mathrm{SFR}}$ for individual groups and the total $\Sigma_{\mathrm{SFR}}$ for the entire region as well as the corresponding cloud gas surface density $\left(\Sigma_{\text {gas }}\right)$ are listed in Table 5. For AFGL 333, the average values of $\Sigma_{\text {gas }}$ and $\Sigma_{\mathrm{SFR}}$ are $\sim 140-160 M_{\odot} \mathrm{pc}^{-2}$ and $\sim 2-3 M_{\odot}$ $\mathrm{Myr}^{-1} \mathrm{pc}^{-2}$, respectively. The above estimates of stellar mass and various parameters are subjected to bias introduced by the unresolved binary/multiple systems. About $75 \%$ of the stars that make up the standard IMF are M-type stars that have a binary fraction of $\sim 20 \%-40 \%$ (Basri \& Reiners 2006; Lada 2006). Assuming 30\% of member stars in AFGL 333 are unresolved binaries, the actual number of forming stars, the star formation efficiency, and SFRs would be increased by about the same factor (Evans et al. 2009).

\section{DISCUSSION}

The W3 giant molecular cloud complex has long been discussed as a classic example of induced or triggered star formation (e.g., Lada et al. 1978; Oey et al. 2005). The expansion of the nearby W4 super bubble may have created the "high density layer (HDL)" of molecular gas at its western periphery. W3 Main, W3 $(\mathrm{OH})$, and AFGL 333 are the three main star-forming sites identified within the HDL. W3 Main 
and $\mathrm{W} 3(\mathrm{OH})$ are located at the edges of the cavity created by the young cluster IC 1795. It has been proposed that the star formation within W3 Main and W3 (OH) are induced by IC 1795 (e.g., Oey et al. 2005; Román-Zúñiga et al. 2015). The recent survey of O- and B-type stars in W3 by Kiminki et al. (2015) found that B stars are widely dispersed across the W3 GMC and they suggest spontaneous star formation started about 8-10 Myr years ago in W3. According to RiveraIngraham et al. (2013), in W3 Main the triggering processes work at local (sub-parsec) scales, with high-mass stars acting to confine and compress material, enhancing the efficiency for the formation of new high-mass stars by making convergent flow. In converging flows, the formation of new stars in clusters can be facilitated for an extended period as the structures will continue drain matter until the matter in their reservoirs get depleted. As pursued by Rivera-Ingraham et al. (2013, 2015), the combined effects of constructive feedback and converging flow could lead to the unique population of high-mass stars and clusters in W3 Main. W3 Main and AFGL 333 are possibly different in terms of the cloud density and dynamics. W3 Main perhaps had an initial high density that resulted in very highmass stars whereas AFGL 333 does not have a density as high as W3 Main.

Using deep NIR and Spitzer data sets, we obtained the census of YSOs within AFGL 333 as well as its cloud structure and mass. In this section, we compare the SFR density and gas density estimated for AFGL 333 with nearby low mass clouds as well as high-mass regions. We also compare these parameters between W3 Main and AFGL 333 and discuss whether the differences between them have any implication due to the various star formation scenarios proposed in these two regions.

\subsection{Comparison of AFGL 333 with Other Star-forming Regions and W3 Main}

The surface density of SFR $\left(\Sigma_{\mathrm{SFR}}\right)$ ranges from 0.1 and 3.4 $M_{\odot} \mathrm{Myr}^{-1} \mathrm{pc}^{-2}$ for a sample of 20 local low-mass star-forming regions (Heiderman et al. 2010). Similarly, Evans et al. (2009) reported values between 0.6 and $3.2 M_{\odot} \mathrm{Myr}^{-1} \mathrm{pc}^{-2}$ for the nearby low-mass Spitzer c2d/GB clouds. Both of those studies have used the NIR extinction map for cloud mass estimation, similar to the method followed in this study. The $\Sigma_{\text {SFR }}$ measured toward AFGL $333\left(\sim 2-3 M_{\odot} \mathrm{Myr}^{-1} \mathrm{pc}^{-2}\right.$; Table 5) is comparable to that of these low-mass regions.

We also compare the $\Sigma_{\text {SFR }}$ of AFGL 333 with the high-mass star-forming regions such as NGC 6334, W43, and IRDC G035.39-00.33 (Motte et al. 2012; Willis et al. 2013). NGC 6334 is located at a similar distance $(\sim 1.6 \mathrm{kpc})$ and the YSO identification and cloud mass estimation from extinction map are calculated in a similar method as in AFGL 333. $\Sigma_{\mathrm{SFR}}$ of NGC 6334 is of $\sim 13.0 M_{\odot} \mathrm{Myr}^{-1} \mathrm{pc}^{-2}$, a factor of $\sim 6$ higher than AFGL 333. For W43 and IRDC G035.39-00.33, $\Sigma_{\text {SFR }}$ is as high as $\sim 10-100 M_{\odot} \mathrm{Myr}^{-1} \mathrm{pc}^{-2}$ (Motte et al. 2003, 2012).

W3 Main and AFGL 333 are part of the same cloud complex and have similar sizes $\left(\sim 0.45 \mathrm{deg}^{2}\right.$, Lada et al. 1978; RiveraIngraham et al. 2013) and ages ( 2-3 Myr, Bik et al. 2012). W3 Main is the most active star-forming region in the entire W3 complex, which contains more than $10 \mathrm{H}$ II regions of various evolutionary status (Tieftrunk et al. 1997; Ojha et al. 2004, 2009) and more than 15 massive stars of spectral type O3V-B0V (Bik et al. 2012) within the central $5.4 \mathrm{pc}^{2}$ area. The central area of W3 Main has a cloud density as high as $\sim 2 \times 10^{23} \mathrm{~cm}^{2}$, with two clumps of masses 1700 and $800 \mathrm{M}_{\odot}$, respectively (Rivera-Ingraham et al. 2013). These values satisfy the conditions for high-mass star formation, which means that W3 Main may continue forming massive stars in the future. Unlike W3 Main, at present, AFGL 333 does not have a column density above the threshold for the formation of massive stars (i.e., $N_{\mathrm{H}_{2}} \sim 1.8 \times 10^{23} \mathrm{~cm}^{2}$; Krumholz \& McKee 2008; Rivera-Ingraham et al. 2013). So far only one massive star of spectral type B0.5 V has been identified within AFGL 333 (Hughes \& Viner 1982).

For W3 Main, the SFE has been estimated as $\sim 44 \%$ by Bik et al. (2012) for an area $\sim 6.5 \mathrm{pc}^{2}$ around the core of W3 Main. However, this is to be considered an upper limit as the cloud mass is estimated from ${ }^{13} \mathrm{CO}$ and $\mathrm{C}^{18} \mathrm{O}$ observations (Dickel 1980; Thronson 1986) where only the dense part of the region would have contributed to the cloud mass estimate. Assuming the age of W3 Main as 2-3 Myr (Bik et al. 2012), the $\Sigma_{\mathrm{SFR}}$ comes out to be $\sim 110-160 M_{\odot} \mathrm{Myr}^{-1} \mathrm{pc}^{-2}$, a factor of $\sim 50$ higher than that of AFGL 333.

In spite of being part of the same giant molecular cloud complex with similar sizes and ages, W3 Main and AFGL 333 differ substantially in their level of star-forming activities. This is similar to the SFR variation observed among the moststudied nearby active star formation sites in the Orion subregions, which includes regions such as ONC, L1630, L1641, etc. These clouds each have masses (a few $\times 10^{4} M_{\odot}$ ) comparable to that of AFGL 333. The SFR in L1630 is known to be a factor of 2-7 lower than that of the nearby L1641, despite having a very similar total reservoir of molecular material (Meyer et al. 2008; Spezzi et al. 2015). One possible explanation is the difference in the spatial distribution of dense gas between the two clouds (Meyer et al. 2008). Similarly, W3 Main has a much larger reservoir of star-forming gas in a compact area, possibly formed by the convergent constructive feedback scenario proposed by Rivera-Ingraham et al. (2013), which causes an enhanced SFR when compared to AFGL 333 (e.g., Lada et al. 2010; Gutermuth et al. 2011). The cloud distribution as well as the star formation activities within W3 Main is similar to that of the other high-mass star-forming regions such as NGC 6334 and W43, whereas AFGL 333 resembles other low-mass star-forming regions.

\subsection{Implications for Triggered Star Formation in AFGL 333}

Observational signposts of triggering in star-forming regions include pillar or cometary-type structures protruding toward the massive stars, YSOs coinciding with bubble rims/shells, age gradients between the YSOs located outside and inside the bright rim clouds, temperature gradients, etc. (e.g., Chauhan et al. 2011; Jose et al. 2013; Pandey et al. 2013). The bright rim cloud BRC 5 is located toward the southeast of AFGL 333 and several short pillars extend into the W4 bubble interior. The winds and radiation emanating from young massive stars of W4 have sculpted these elongated elephant trunks out of the surrounding molecular material. Based on an optical photometric analysis, Panwar et al. (2014) reported that the YSOs located within the bright rim of BRC 5 are younger than those YSOs outside the rim. Similarly, the dust temperature map from Herschel observations shows that the temperature is high $(\sim 22 \mathrm{~K})$ near the ionization front at BRC 5, and is relatively low $(<14 \mathrm{~K})$ near the ridge (Rivera-Ingraham et al. 2013). The higher temperature at the ionization front indicates that it has been heated by the strong radiation from W4. The above 
examples are some of the classical signatures of triggered star formation that are seen at the eastern edge of AFGL 333.

Triggered star formation can be defined in many ways, such as a temporary or long-term increase in the SFR, an increase in the final star formation efficiency, or an increase in the total final number of stars formed (Dale et al. 2015). Our observational analysis shows that the star formation efficiency and rate within AFGL 333 are comparable to nearby low-mass star-forming regions. Stellar feedback has apparently not enhanced the star formation efficiency of AFGL 333. If the massive OB stars of W4 had strong influence on AFGL 333, then the closer region (AFGL 333 Main) should be more affected than the distant region (AFGL 333 NW2). However, we find almost similar ages, star formation efficiencies, and SFRs for the sub-clusters of AFGL 333.

Our analysis suggests that sequential star formation, which one would expect for a cloud under the influence of external ionizing photons, is not the prime mechanism of star formation in AFGL 333. Star formation influenced by external feedback does not seem to have propagated throughout the cloud within AFGL 333. Although individual YSOs at the tips of the small clouds (e.g., BRC 5, pillars) might be triggered, their contribution to the overall star formation efficiency and rate of the entire cloud is low. The star formation in these subclusters could have started due to the primordial cloud collapse. The ionization and shock front of the W4 bubble may have stalled at the east of AFGL 333, giving the impression of triggering.

We remind the readers that the star formation parameters of AFGL 333 are the average properties of the three major stellar groups (see Section 5.1), which comprise sub-clusters of various evolutionary status, environments, and external conditions. Due to the limited sensitivity and spatial resolution of the data sets in this study, we do not segregate the different populations within AFGL 333 Main. Hence we are unable to detect any local triggering process acting in scales of $\sim 1.5 \mathrm{pc}$ or less. In conclusion, W4 $\mathrm{H}$ in region appears to have little or no effect on the overall, averaged star formation activity within AFGL 333, except that for the easternmost regions that associated with BRC 5.

Disentangling triggered star formation from spontaneous star formation requires precise determination of proper motion and ages of individual sources (Dale et al. 2015). Even if we assume that some triggering is going on at the eastern side of the AFGL 333 cloud, the overall effect of feedback from W4 on the properties of the entire AFGL 333 cloud is low. Numerical simulations by Dale \& Bonnell (2012) also show that stellar feedback may simultaneously enhance or suppress star formation and may not have a strong effect on the overall star formation efficiency. Detailed observational studies of a large sample of star-forming regions are needed to strengthen the hypothesis that feedback does not measurably affect the global SFR and efficiency.

\section{SUMMARY}

The W3 giant molecular cloud complex is one of the most active massive star-forming regions in the outer Galaxy. W3 Main, W3 (OH), and AFGL 333 are the major sub-regions within W3. This complex has been subject to numerous investigations as it is an excellent laboratory for studying the feedback effect from massive stars of the nearby W4 super bubble. The low mass stellar content of AFGL 333 was poorly explored until this study.

We analyzed the deep $J H K_{s}$ observations complimented with Spitzer IRAC and MIPS observations to unravel the low-mass stellar population as well as to understand the cloud structure and star formation activity within AFGL 333. Based on the NIR and mid-IR colors, we identified 812 candidate YSOs in this region, of which 99 are classified as Class I and 713 as Class II sources. The survey is complete down to $\sim 0.2 \mathrm{M}_{\odot}$. This survey increases the census of YSO members of the region by a factor $>3$ compared to previous studies. The spatial distribution and the stellar density analysis shows that a majority of the candidate YSOs are located mainly within three stellar groups, called AFGL 333 Main, AFGL 333 NW1, and AFGL 333 NW2. The disk fraction estimated within three stellar groups are $\sim 50 \%-60 \%$.

Using NIR data as well as CO-based column density map, extinction maps across AFGL 333 is constructed in order to understand the cloud structure as well as to estimate the cloud mass of the region. Combining the stellar mass with cloud mass, the average star formation efficiency of the region has been estimated as $\sim 4.5 \%$ and SFR as $\sim 130-190 M_{\odot} \mathrm{Myr}^{-1}$. The SFR density $\left(\Sigma_{\text {SFR }}\right)$ measured within AFGL 333 is $\sim 2-3 M_{\odot} \mathrm{Myr}^{-1} \mathrm{pc}^{-2}$.

We compared the star formation activity of AFGL 333 with that of nearby low-mass and high-mass star-forming regions as well as with W3 Main. The SFR density of AFGL 333 is similar to the nearby low mass star-forming regions but is a factor of $\sim 50$ lower than that of W3 Main. Currently AFGL 333 is not dense enough to form massive stars. On the other hand, the star formation activity within W3 Main is comparable to other high-mass star-forming regions of the Milky Way and the region is still dense enough to form many more massive stars in future.

Though we observe some of the classical signs of triggering such as pillars, bright rim clouds, etc. toward the eastern edge of AFGL 333, we find no evidence to suggest that stellar feedback has influenced the global star formation activity within AFGL 333. The star formation activity in AFGL 333 and W3 Main are different most likely due to the difference in the gas density within them as well as due to the differences in the feedback mechanisms in these two regions. However, detailed studies of a large sample of externally influenced regions are essential in order to quantify this statement.

We are grateful to the anonymous referee for his/her constructive comments which have helped us to improve the scientific contents of the paper. We express our sincere gratitude to Robert Swaters for his help with NEWFIRM data reduction and Alana Rivera-Ingraham for sharing the column density map. This work is supported by a Youth Qianren grant to G.J.H. and general grant \# 11473005 awarded by the National Science Foundation of China. This project is also based upon work supported by the National Science Foundation, USA, under Astronomy and Astrophysics Research Grant AST-0907980 to J.S.K. The observations reported here were obtained at the Kitt Peak National Observatory, National Optical Astronomy Observatory, which is operated by the Association of Universities for Research in Astronomy (AURA), Inc., under cooperative agreement with the National Science Foundation. This research has made use of the SIMBAD database (operated at CDS, Strasbourg, France), 
Two Micron All Sky Survey (a joint project of the University of Massachusetts and the Infrared Processing and Analysis Center/California Institute of Technology, funded by NASA and NSF), and archival data obtained with the Spitzer Space Telescope (operated by the Jet Propulsion Laboratory, California Institute of Technology under a contract with NASA).

\section{REFERENCES}

Allen, T. S., Pipher, J. L., Gutermuth, R. A., et al. 2008, ApJ, 675, 491 Basri, G., \& Reiners, A. 2006, AJ, 132, 663

Beerer, I. M., Koenig, X. P., Hora, J. L., et al. 2010, ApJ, 720, 679

Bica, E., Dutra, C. M., \& Barbuy, B. 2003, A\&A, 397, 177

Bieging, J. H., \& Peters, W. L. 2011, ApJS, 196, 18

Bik, A., Henning, T., Stolte, A., et al. 2012, ApJ, 744, 87

Billot, N., Noriega-Crespo, A., Carey, S., et al. 2010, ApJ, 712, 797

Bisbas, T. G., Wünsch, R., Whitworth, A. P., Hubber, D. A., \& Walch, S. 2011, ApJ, 736, 142

Black, J. H., \& van Dishoeck, E. F. 1987, ApJ, 322, 412

Bohlin, R. C., Savage, B. D., \& Drake, J. F. 1978, ApJ, 224, 132

Bressan, A., Marigo, P., Girardi, L., et al. 2012, MNRAS, 427, 127

Cardelli, J. A., Clayton, G. C., \& Mathis, J. S. 1989, ApJ, 345, 245

Casertano, S., \& Hut, P. 1985, ApJ, 298, 80

Chapman, N. L., Mundy, L. G., Lai, S.-P., \& Evans, N. J., II 2009, ApJ, 690, 496

Chauhan, N., Ogura, K., Pandey, A. K., Samal, M. R., \& Bhatt, B. C. 2011, PASJ, 63, 795

Chavarría, L. A., Allen, L. E., Hora, J. L., Brunt, C. M., \& Fazio, G. G. 2008 , ApJ, 682, 445

Dale, J. E., \& Bonnell, I. A. 2012, MNRAS, 422, 1352

Dale, J. E., Ercolano, B., \& Bonnell, I. A. 2013, MNRAS, 430, 234

Dale, J. E., Haworth, T. J., \& Bressert, E. 2015, MNRAS, 450, 1199

Dickel, H. R. 1980, ApJ, 238, 829

Draine, B. T. 2003, ARA\&A, 41, 241

Elmegreen, B. G., \& Lada, C. J. 1977, ApJ, 214, 725

Evans, N. J., II, Dunham, M. M., Jørgensen, J. K., et al. 2009, ApJS, 181, 321

Fang, M., Kim, J. S., van Boekel, R., et al. 2013, ApJS, 207, 5

Fang, M., van Boekel, R., King, R. R., et al. 2012, A\&A, 539, A119

Fazio, G. G., Hora, J. L., Allen, L. E., et al. 2004, ApJS, 154, 10

Flaherty, K. M., Pipher, J. L., Megeath, S. T., et al. 2007, ApJ, 663, 1069

Green, G. M., Schlafly, E. F., Finkbeiner, D. P., et al. 2015, ApJ, 810, 25

Gutermuth, R. A., Megeath, S. T., Myers, P. C., et al. 2009, ApJS, 184, 18

Gutermuth, R. A., Megeath, S. T., Pipher, J. L., et al. 2005, ApJ, 632, 397

Gutermuth, R. A., Pipher, J. L., Megeath, S. T., et al. 2011, ApJ, 739, 84

Hachisuka, K., Brunthaler, A., Menten, K. M., et al. 2006, ApJ, 645, 337

Haisch, K. E., Jr., Lada, E. A., \& Lada, C. J. 2001, ApJL, 553, L153

Heiderman, A., Evans, N. J., II, Allen, L. E., Huard, T., \& Heyer, M. 2010, ApJ, 723, 1019

Hernández, J., Hartmann, L., Calvet, N., et al. 2008, ApJ, 686, 1195

Higuchi, A. E., Kurono, Y., Naoi, T., et al. 2013, ApJ, 765, 101

Hughes, V. A., \& Viner, M. R. 1982, AJ, 87, 685

Indebetouw, R., Mathis, J. S., Babler, B. L., et al. 2005, ApJ, 619, 931

Jose, J., Pandey, A. K., Ogura, K., et al. 2012, MNRAS, 424, 2486

Jose, J., Pandey, A. K., Samal, M. R., et al. 2013, MNRAS, 432, 3445

Kauffmann, J., Bertoldi, F., Bourke, T. L., Evans, N. J., II, \& Lee, C. W. 2008, A\&A, 487, 993

Kerton, C. R., Arvidsson, K., Knee, L. B. G., \& Brunt, C. 2008, MNRAS, 385, 995

Kim, H.-J., Koo, B.-C., \& Davis, C. J. 2015, ApJ, 802, 59

Kiminki, M. M., Kim, J. S., Bagley, M. B., Sherry, W. H., \& Rieke, G. H. 2015, ApJ, 813, 42

Koenig, X. P., Allen, L. E., Gutermuth, R. A., et al. 2008, ApJ, 688, 1142

Kroupa, P. 2002, Sci, 295, 82

Krumholz, M. R., \& McKee, C. F. 2008, Natur, 451, 1082

Kulesa, C. A., Hungerford, A. L., Walker, C. K., Zhang, X., \& Lane, A. P. 2005, ApJ, 625, 194
Lada, C. J. 2006, ApJL, 640, L63

Lada, C. J., Elmegreen, B. G., Cong, H.-I., \& Thaddeus, P. 1978, ApJL, 226, L39

Lada, C. J., Lombardi, M., \& Alves, J. F. 2010, ApJ, 724, 687

Lada, C. J., \& Wilking, B. A. 1984, ApJ, 287, 610

Massi, F., Giannetti, A., Di Carlo, E., et al. 2015, A\&A, 573, A95

Mathis, J. S. 1990, ARA\&A, 28, 37

Meyer, M. R., Calvet, N., \& Hillenbrand, L. A. 1997, AJ, 114, 288

Meyer, M. R., Flaherty, K., Levine, J. L., et al. 2008, in in Handbook of Star Forming Regions, Volume I: The Northern Sky ASP Monograph Publications, Vol. 4, ed. B. Reipurth (San Francisco, CA: ASP), 664

Milam, S. N., Savage, C., Brewster, M. A., Ziurys, L. M., \& Wyckoff, S. 2005, ApJ, 634, 1126

Moore, T. J. T., Bretherton, D. E., Fujiyoshi, T., et al. 2007, MNRAS, 379, 663

Motte, F., Bontemps, S., Hennemann, M., et al. 2012, in SF2A-2012: Proc. Annual Meeting of the French Society of Astronomy and Astrophysics, ed. S. Boissier et al., 45

Motte, F., Schilke, P., \& Lis, D. C. 2003, ApJ, 582, 277

Myers, P. C. 2012, ApJ, 752, 9

Myers, P. C., Dame, T. M., Thaddeus, P., et al. 1986, ApJ, 301, 398

Oey, M. S., Watson, A. M., Kern, K., \& Walth, G. L. 2005, AJ, 129, 393

Ojha, D. K., Tamura, M., Nakajima, Y., et al. 2004, ApJ, 608, 797

Ojha, D. K., Tamura, M., Nakajima, Y., et al. 2009, ApJ, 693, 634

Pandey, A. K., Eswaraiah, C., Sharma, S., et al. 2013, ApJ, 764, 172

Panwar, N., Chen, W. P., Pandey, A. K., et al. 2014, MNRAS, 443, 1614

Patten, B. M., Stauffer, J. R., Burrows, A., et al. 2006, ApJ, 651, 502

Pineda, J. L., Goldsmith, P. F., Chapman, N., et al. 2010, ApJ, 721, 686

Polychroni, D., Moore, T. J. T., \& Allsopp, J. 2012, MNRAS, 422, 2992

Povich, M. S., Kuhn, M. A., Getman, K. V., et al. 2013, ApJS, 209, 31

Probst, R. G., Gaughan, N., Abraham, M., et al. 2004, Proc. SPIE, 5492, 1716

Rieke, G. H., Young, E. T., Engelbracht, C. W., et al. 2004, ApJS, 154, 25

Rivera-Ingraham, A., Martin, P. G., Polychroni, D., \& Moore, T. J. T. 2011, ApJ, 743, 39

Rivera-Ingraham, A., Martin, P. G., Polychroni, D., et al. 2013, ApJ, 766, 85

Rivera-Ingraham, A., Martin, P. G., Polychroni, D., et al. 2015, ApJ, 809, 81

Robin, A. C., Reylé, C., Derrière, S., \& Picaud, S. 2003, A\&A, 409, 523

Robitaille, T. P., Meade, M. R., Babler, B. L., et al. 2008, AJ, 136, 2413

Román-Zúñiga, C. G., Ybarra, J. E., Megías, G. D., et al. 2015, AJ, 150, 80

Rowan-Robinson, M., Gonzalez-Solares, E., Vaccari, M., \& Marchetti, L. 2013, MNRAS, 428, 1958

Ruch, G. T., Jones, T. J., Woodward, C. E., et al. 2007, ApJ, 654, 338

Sakai, T., Oka, T., \& Yamamoto, S. 2006, ApJ, 649, 268

Samal, M. R., Ojha, D. K., Jose, J., et al. 2015, A\&A, 581, A5

Samal, M. R., Zavagno, A., Deharveng, L., et al. 2014, A\&A, 566, A122

Skrutskie, M. F., Cutri, R. M., Stiening, R., et al. 2006, AJ, 131, 1163

Soderblom, D. R., Hillenbrand, L. A., Jeffries, R. D., Mamajek, E. E., \& Naylor, T. 2014, in Protostars and Planets VI, Observations of Jets and Outflows from Young Stars, ed. H. Beuther, R. S. Klessen, C. P. Dullemond, \& T. Henning (Tuscon, AZ: Univ. Arizona Press), 219

Spezzi, L., Petr-Gotzens, M. G., Alcalá, J. M., et al. 2015, A\&A, 581, A140

Stetson, P. B. 1987, PASP, 99, 191

Sugitani, K., Fukui, Y., \& Ogura, K. 1991, ApJS, 77, 59

Swaters, R. A., Valdes, F., \& Dickinson, M. E. 2009, in ASP Conf. Ser. 411, Astronomical Data Analysis Software and Systems XVIII, ed. D. A. Bohlender, D. Durand, \& P. Dowler (San Francisco, CA: ASP), 506 Thronson, H. A., Jr. 1986, ApJ, 306, 160

Tieftrunk, A. R., Gaume, R. A., Claussen, M. J., Wilson, T. L., \& Johnston, K. J. 1997, A\&A, 318, 931

van Dishoeck, E. F., \& Black, J. H. 1988, ApJ, 334, 771

Walch, S., Whitworth, A. P., Bisbas, T. G., Wünsch, R., \& Hubber, D. A. 2013, MNRAS, 435, 917

Werner, M. W., Roellig, T. L., Low, F. J., et al. 2004, ApJS, 154, 1

Whitney, B. A., Sewilo, M., Indebetouw, R., et al. 2008, AJ, 136, 18

Williams, J. P., \& Cieza, L. A. 2011, ARA\&A, 49, 67

Willis, S., Marengo, M., Allen, L., et al. 2013, ApJ, 778, 96

Wu, J., Evans, N. J., II, Shirley, Y. L., \& Knez, C. 2010, ApJS, 188, 313

Xu, Y., Reid, M. J., Zheng, X. W., \& Menten, K. M. 2006, Sci, 311, 54

Zeidler, P., Preibisch, T., Ratzka, T., Roccatagliata, V., \& Petr-Gotzens, M. G. 2016, A\&A, 585, A49 